Invited lectures at the NATO ASI Structure Formation in the Universe

Cambridge, August 1999

\title{
CLUSTERING OF MASS AND GALAXIES
}

\author{
J.A. PEACOCK \\ Institute for Astronomy, University of Edinburgh \\ Royal Observatory, Edinburgh EH9 3HJ, UK
}

\begin{abstract}
These lectures cover various aspects of the statistical description of cosmological density fields. Observationally, this consists of the point process defined by galaxies, and the challenge is to relate this to the continuous density field generated by gravitational instability in dark matter. The main topics discussed are (1) nonlinear structure in CDM models; (2) statistical measures of clustering; (3) redshift-space distortions; (4) smallscale clustering and bias. The overall message is optimistic, in that simple assumptions for where galaxies should form in the mass density field allow one to understand the systematic differences between galaxy data and the predictions of CDM models.
\end{abstract}

\section{Preamble}

The subject of large-scale structure is in a period of very rapid development. For many years, this term would have meant only one thing: the distribution of galaxies. However, we are increasingly able to probe the primordial fluctuations through the CMB, so that the problem of galaxy formation and clustering is now only one aspect of the general picture of structure formation. The rationale for studying the large-scale distribution of galaxies is therefore altering. Ten years ago, we were happy to produce samples based on a rather sparse random sampling of the galaxy distribution, with the main aim of tying down statistics such as the large-scale power spectrum of number-density fluctuations. A major goal of the subject remains the measurement of the fluctuation spectrum for wavelengths $\gtrsim 100 \mathrm{Mpc}$, and the demonstration that this agrees in shape with what can be inferred from the CMB. Nevertheless, we are now increasingly interested in studying the pattern of galaxies with the highest possible fidelity - demanding deep, fully-sampled surveys of the local universe. Such studies will tell us 
much about the processes by which galaxies formed and evolved within the distribution of dark matter. The aim of these lectures is therefore to look both backwards and forwards: reviewing the foundations of the subject and looking forward to the future issues.

\section{The CDM family album}

\subsection{THE LINEAR SPECTRUM}

The basic picture of inflationary models (but also of cosmology before inflation) is of a primordial power-law spectrum, written dimensionlessly as the logarithmic contribution to the fractional density variance, $\sigma^{2}$ :

$$
\Delta^{2}(k)=\frac{d \sigma^{2}}{d \ln k} \propto k^{3+n},
$$

where $n$ stands for $n_{\mathrm{S}}$ hereafter. This undergoes linear growth

$$
\delta_{k}(a)=\delta_{k}\left(a_{0}\right)\left[\frac{D(a)}{D\left(a_{0}\right)}\right] T_{k},
$$

where the linear growth law is

$$
D(a)=a g[\Omega(a)]
$$

in the matter era, and the growth suppression for low $\Omega$ is

$$
\begin{aligned}
g(\Omega) & \simeq \Omega^{0.65} \text { (open) } \\
& \simeq \Omega^{0.23} \text { (flat) }
\end{aligned}
$$

The transfer function $T_{k}$ depends on the dark-matter content as shown in figure 1.

Note the baryonic oscillations in figure 1; these can be significant even in CDM-dominated models when working with high-precision data. Eisenstein \& $\mathrm{Hu}$ (1998) are to be congratulated for their impressive persistence in finding an accurate fitting formula that describes these wiggles. This is invaluable for carrying out a search of a large parameter space.

The state of the linear-theory spectrum after these modifications is illustrated in figure 2. The primordial power-law spectrum is reduced at large $k$, by an amount that depends on both the quantity of dark matter and its nature. Generally the bend in the spectrum occurs near $1 / k$ of order the horizon size at matter-radiation equality, $\propto\left(\Omega h^{2}\right)^{-1}$. For a pure CDM universe, with scale-invariant initial fluctuations $(n=1)$, the observed spectrum depends only on two parameters. One is the shape $\Gamma=\Omega h$, and the

other is a normalization. On the shape front, a government health warning 


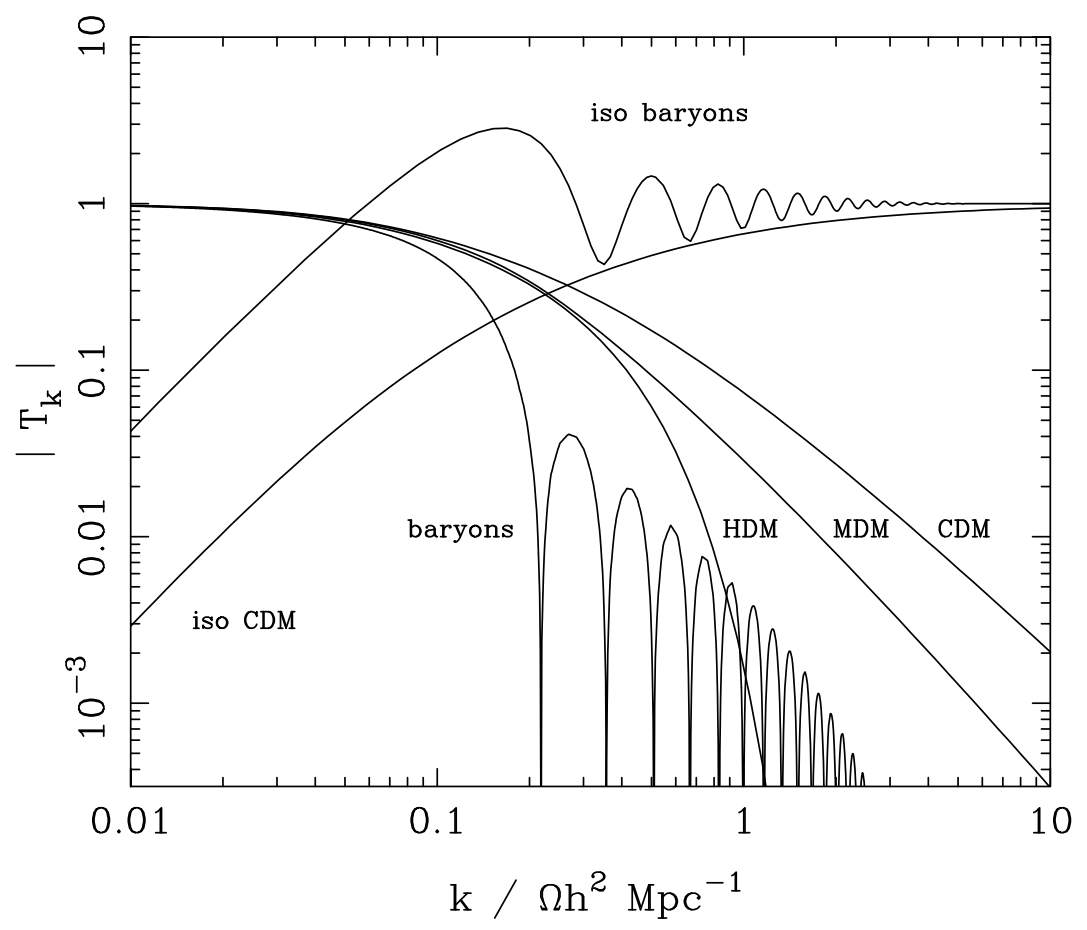

Figure 1. Transfer functions for various dark-matter models. The scaling with $\Omega h^{2}$ is exact only for the zero-baryon models; the baryon results are scaled from the particular case $\Omega_{\mathrm{B}}=1, h=1 / 2$.

is needed, as follows. It has been quite common to take $\Gamma$-based fits to observations as indicating a measurement of $\Omega h$, but there are three reasons why this may give incorrect answers:

(1) The dark matter may not be CDM. An admixture of HDM will damp the spectrum more, mimicking a lower CDM density.

(2) Even in a CDM-dominated universe, baryons can have a significant effect, making $\Gamma$ lower than $\Omega h$. An approximate formula for this is given in figure 2 (Peacock \& Dodds 1994; Sugiyama 1995).

(3) The strongest (and most-ignored) effect is tilt: if $n \neq 1$, then even in a pure CDM universe a $\Gamma$-model fit to the spectrum will give a badly incorrect estimate of the density (the change in $\Omega h$ is roughly $0.3(n-1)$; Peacock \& Dodds 1994).

\subsection{NORMALIZATION}

The other parameter is the normalization. This can be set at a number of points. The COBE normalization comes from large angle CMB anisotropies, 


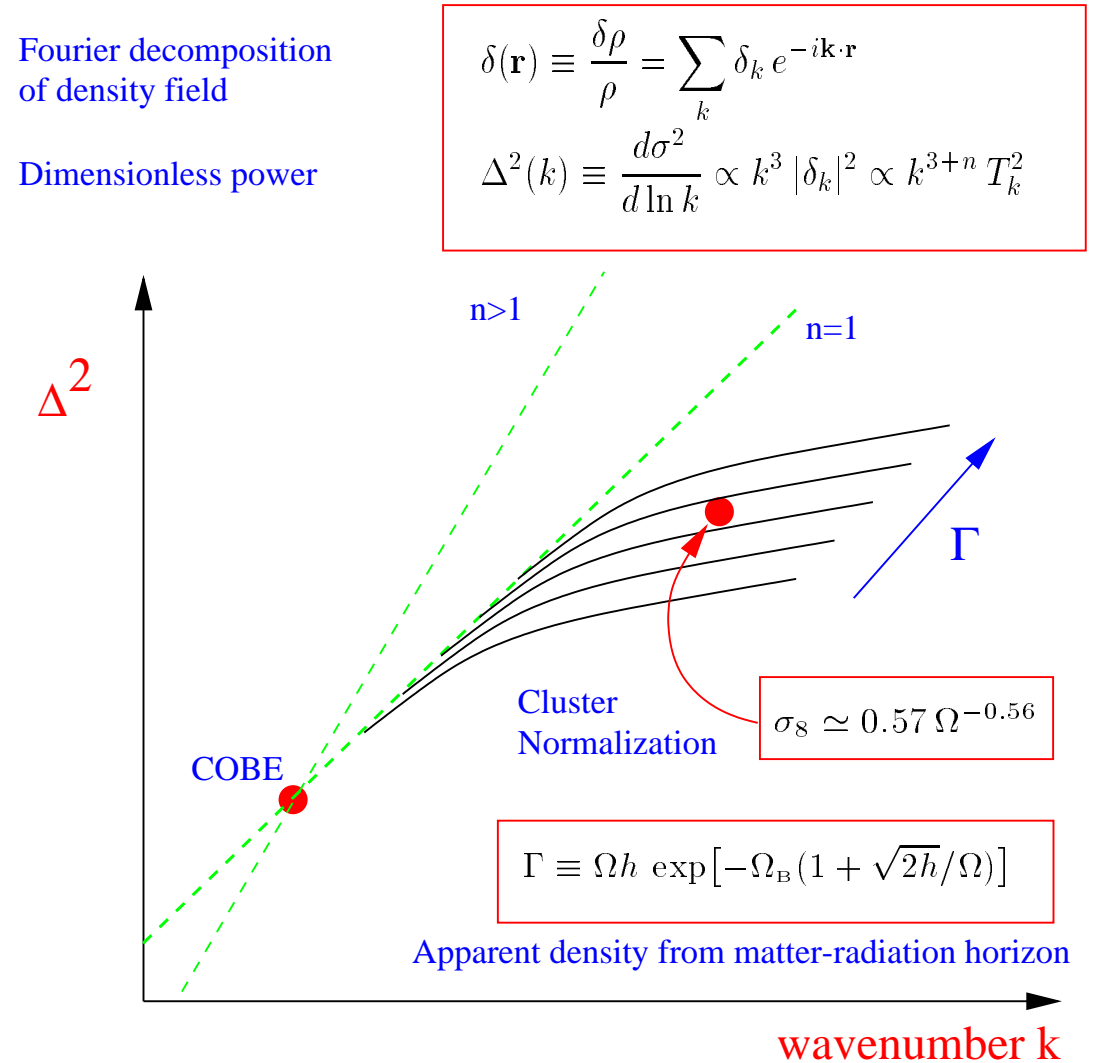

$$
\sigma_{8}^{2}=\Delta^{2}\left(k_{\mathrm{eff}}\right), \quad k_{\mathrm{eff}} / h \mathrm{Mpc}^{-1}=0.172+0.011[\ln (\Gamma / 0.34)]^{2}
$$

Figure 2. This figure illustrates how the primordial power spectrum is modified as a function of density in a CDM model. For a given tilt, it is always possible to choose a density that satisfies both the COBE and cluster normalizations.

and is sensitive to the power spectrum at $k \simeq 10^{-3} h \mathrm{Mpc}^{-1}$. The alternative is to set the normalization near the quasilinear scale, using the abundance of rich clusters. Many authors have tried this calculation, and there is good agreement on the answer:

$$
\sigma_{8} \simeq(0.5-0.6) \Omega_{m}^{-0.6} .
$$

(White, Efstathiou \& Frenk 1993; Eke et al. 1996; Viana \& Liddle 1996). In many ways, this is the most sensible normalization to use for LSS studies, since it does not rely on an extrapolation from larger scales. 


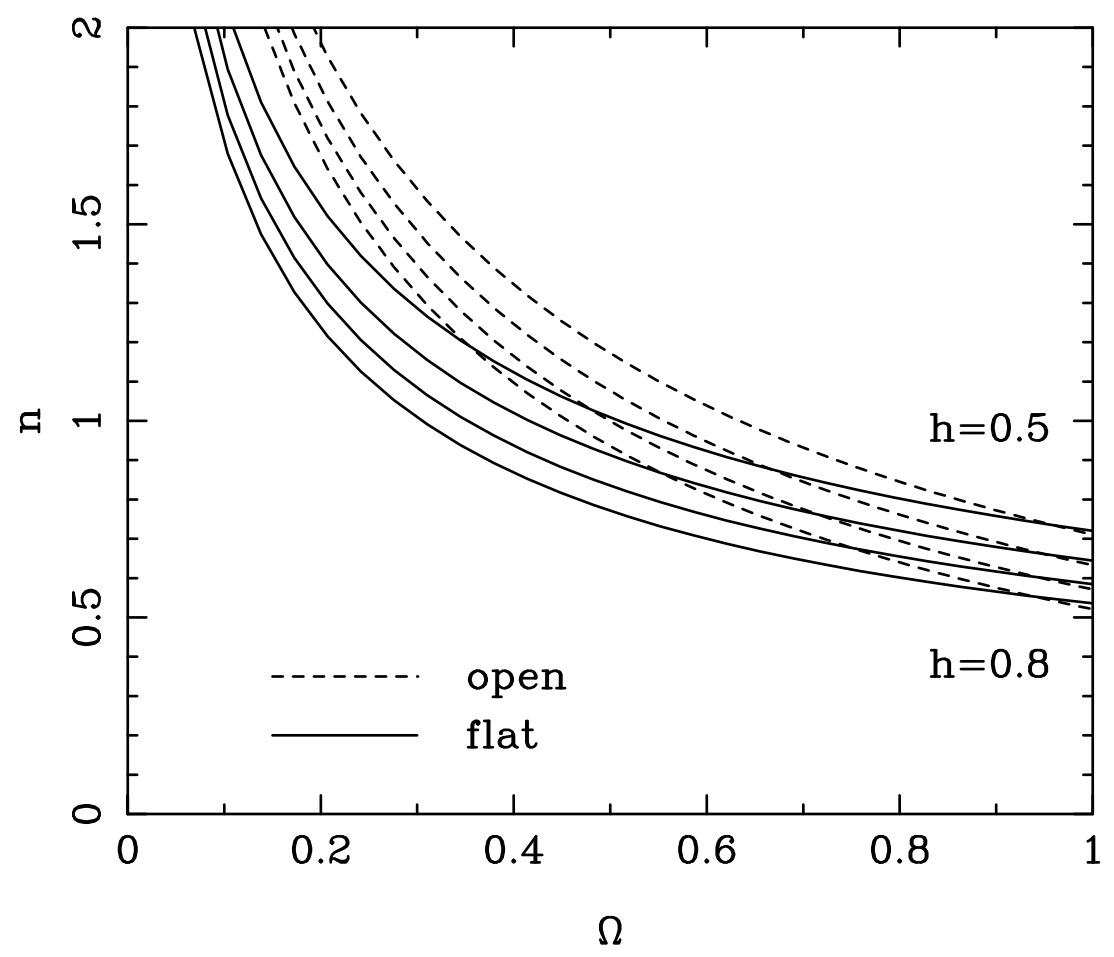

Figure 3. For $10 \%$ baryons, the value of $n$ needed to reconcile COBE and the cluster normalization in CDM models.

Within the CDM model, it is always possible to satisfy both these normalization constraints, by appropriate choice of $\Gamma$ and $n$. This is illustrated in figure 3. Note that vacuum energy affects the answer; for reasonable values of $h$ and reasonable baryon content, flat models require $\Omega_{m} \simeq 0.3$, whereas open models require $\Omega_{m} \simeq 0.5$.

\subsection{THE NONLINEAR SPECTRUM}

On smaller scales $(k \gtrsim 0.1)$, nonlinear effects become important. These are relatively well understood so far as they affect the power spectrum of the mass (e.g. Hamilton et al. 1991; Jain, Mo \& White 1995; Peacock \& Dodds 1996). Based on a fitting formula for the similarity solution governing the evolution of scale-free initial conditions, it is possible to predict the evolved spectrum in CDM universes to a few per cent precision (e.g. Jenkins et al. 1998).

These methods can cope with most smoothly-varying power spectra, but they break down for models with a large baryon content. Figure 1 shows 

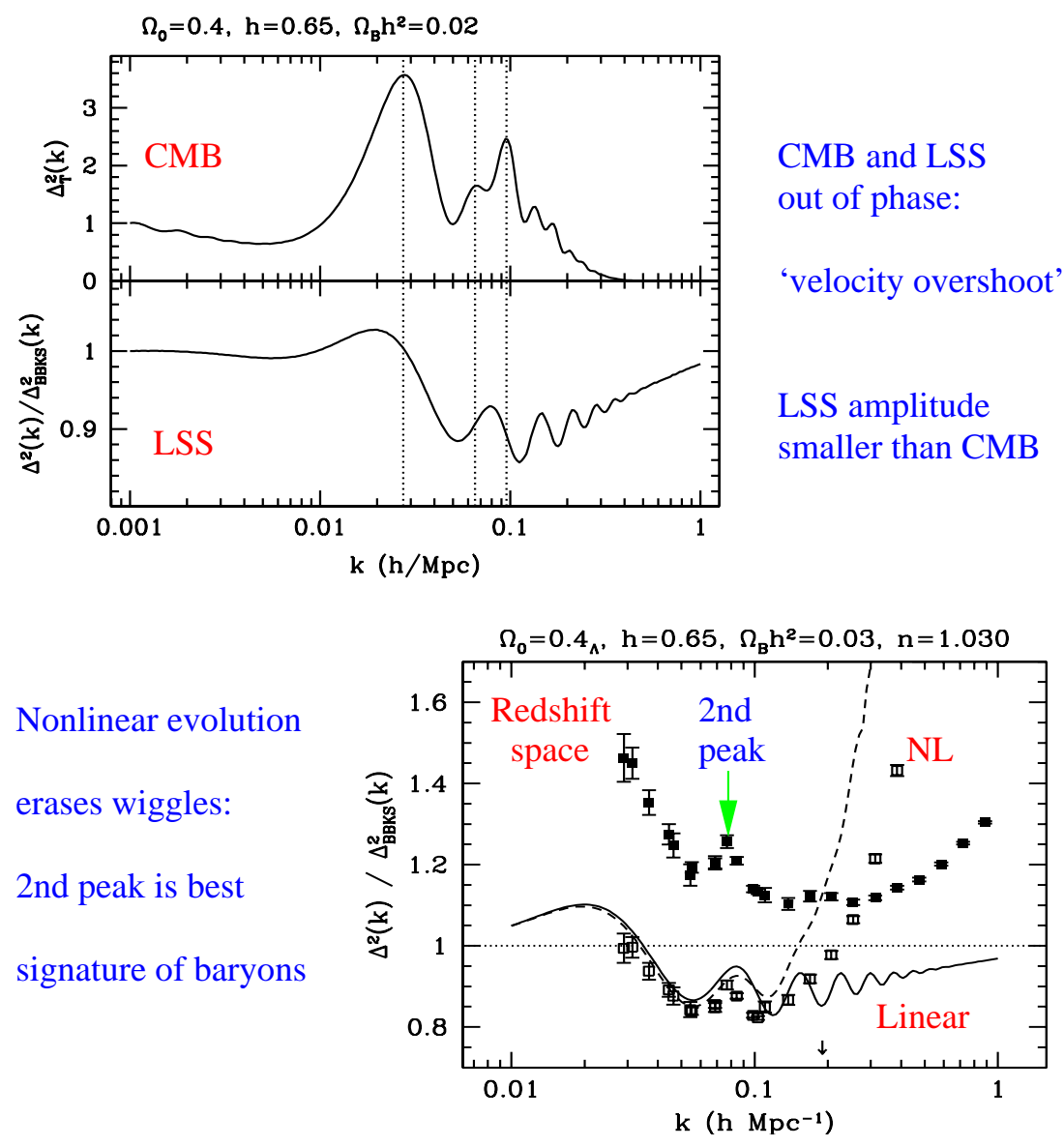

Figure 4. Baryonic fluctuations in the spectrum can become significant for high-precision measurements. Although such features are much less important in the density spectrum than in the CMB (first panel), the order $10 \%$ modulation of the power is potentially detectable. However, nonlinear evolution has the effect of damping all beyond the second peak. This second feature is relatively narrow, and can serve as a clear proof of the past existence of oscillations in the baryon-photon fluid (Meiksin, White \& Peacock 1999).

that rather large oscillatory features would be expected if the universe was baryon dominated. The lack of observational evidence for such features is one reason for believing that the universe might be dominated by collisionless nonbaryonic matter (consistent with primordial nucleosynthesis if $\Omega_{m} \gtrsim 0.1$.

Nevertheless, baryonic fluctuations in the spectrum can become significant for high-precision measurements. Figure 4 shows that order $10 \%$ modulation of the power may be expected in realistic baryonic models (Eisenstein \& Hu 1998; Goldberg \& Strauss 1998). Most of these features are 
however removed by nonlinear evolution. The highest- $k$ feature to survive is usually the second peak, which almost always lies near $k=0.05 \mathrm{Mpc}^{-1}$ (no $h$, for a change). This feature is relatively narrow, and can serve as a clear proof of the past existence of baryonic oscillations in forming the mass distribution (Meiksin, White \& Peacock 1999). However, figure 4 emphasizes that the easiest way of detecting the presence of baryons is likely to be through the CMB spectrum. The oscillations have a much larger "visibility' there, because the small-scale CMB anisotropies come directly from the coupled radiation-baryon fluid, rather than the small-scale dark matter perturbations.

\section{Statistics}

Statistical measures of the cosmological density field relate to properties of the dimensionless density perturbation field

$$
\delta(\mathbf{x}) \equiv \frac{\rho(\mathbf{x})-\langle\rho\rangle}{\langle\rho\rangle},
$$

although $\delta$ need not be assumed to be small.

\subsection{CORRELATION FUNCTIONS}

The simplest measure is the autocorrelation function of the density perturbation

$$
\xi_{\mathrm{A}}(\mathbf{r}) \equiv\langle\delta(\mathbf{x}) \delta(\mathbf{x}+\mathbf{r})\rangle
$$

This is a straightforward statistical measure that can also be computed for the dark-matter distribution in $N$-body simulations. Formally, the averaging operator here is an ensemble average, but one generally appeals to the ergodic nature of the density field to replace this with a volume average.

However, galaxies are a point process, so what astronomers can measure in practice is the two-point correlation function, which gives the excess probability for finding a neighbour a distance $r$ from a given galaxy. By regarding this as the probability of finding a pair with one object in each of the volume elements $d V_{1}$ and $d V_{2}$,

$$
d P=\rho_{0}^{2}\left[1+\xi_{2}(r)\right] d V_{1} d V_{2} .
$$

Is it true that $\xi_{\mathrm{A}}(r)=\xi_{2}(r)$ ? Life would certainly be simple if so, and much work on large-scale structure has implicitly assumed the Poisson clustering hypothesis, in which galaxies are assumed to be sampled at random from some continuous underlying density field. Many of the puzzles in the field 
can however be traced to the fact that this hypothesis is probably false, as discussed below.

A related quantity is the cross-correlation function. Here, one considers two different classes of object ( $a$ and $b$, say), and the cross-correlation function $\xi_{a b}$ is defined as the (symmetric) probability of finding a pair in which $d V_{1}$ is occupied by an object from the first catalogue and $d V_{2}$ by one from the second. Both cross- and auto-correlation functions are readily extended to higher orders and considerations of $n$-tuples of points in a given geometry.

\subsection{FOURIER SPACE}

For the Fourier counterpart of this analysis, we assume that the field is periodic within some box of side $L$, and expand as a Fourier series:

$$
\delta(\mathbf{x})=\sum \delta_{\mathbf{k}} e^{-i \mathbf{k} \cdot \mathbf{x}} .
$$

For a real field, $\delta_{\mathbf{k}}(-\mathbf{k})=\delta_{\mathbf{k}}^{*}(\mathbf{k})$. Using this definition in the correlation function, most cross terms integrate to zero through the periodic boundary conditions, giving

$$
\xi(\mathbf{r})=\frac{V}{(2 \pi)^{3}} \int\left|\delta_{\mathbf{k}}\right|^{2} e^{-i \mathbf{k} \cdot \mathbf{r}} d^{3} k .
$$

In short, the correlation function is the Fourier transform of the power spectrum.

We shall usually express the power spectrum in dimensionless form, as the variance per $\ln k\left(\Delta^{2}(k)=d\left\langle\delta^{2}\right\rangle / d \ln k \propto k^{3} P[k]\right)$ :

$$
\Delta^{2}(k) \equiv \frac{V}{(2 \pi)^{3}} 4 \pi k^{3} P(k)=\frac{2}{\pi} k^{3} \int_{0}^{\infty} \xi(r) \frac{\sin k r}{k r} r^{2} d r .
$$

This gives a more easily visualizable meaning to the power spectrum than does the quantity $\operatorname{VP}(k)$, which has dimensions of volume: $\Delta^{2}(k)=1$ means that there are order-unity density fluctuations from modes in the logarithmic bin around wavenumber $k . \Delta^{2}(k)$ is therefore the natural choice for a Fourier-space counterpart to the dimensionless quantity $\xi(r)$.

In the days before inflation, the primordial power spectrum was chosen by hand, and the minimal assumption was a featureless power law:

$$
\left\langle\left|\delta_{k}\right|^{2}\right\rangle \equiv P(k) \propto k^{n}
$$

The index $n$ governs the balance between large- and small-scale power. Similarly, a power-law spectrum implies a power-law correlation function. 
If $\xi(r)=\left(r / r_{0}\right)^{-\gamma}$, with $\gamma=n+3$, the corresponding 3D power spectrum is

$$
\Delta^{2}(k)=\frac{2}{\pi}\left(k r_{0}\right)^{\gamma} \Gamma(2-\gamma) \sin \frac{(2-\gamma) \pi}{2} \equiv \beta\left(k r_{0}\right)^{\gamma}
$$

$\left(=0.903\left(k r_{0}\right)^{1.8}\right.$ if $\left.\gamma=1.8\right)$. This expression is only valid for $n<0(\gamma<3)$; for larger values of $n, \xi$ must become negative at large $r$ (because $P(0)$ must vanish, implying $\left.\int_{0}^{\infty} \xi(r) r^{2} d r=0\right)$. A cutoff in the spectrum at large $k$ is needed to obtain physically sensible results.

The most interesting value of $n$ is the scale-invariant spectrum, $n=1$, i.e. $\Delta^{2} \propto k^{4}$. To see how the name arises, consider a perturbation $\delta \Phi$ in the gravitational potential:

$$
\nabla^{2} \delta \Phi=4 \pi G \rho_{0} \delta \quad \Rightarrow \quad \delta \Phi_{k}=-4 \pi G \rho_{0} \delta_{k} / k^{2} .
$$

The two powers of $k$ pulled down by $\nabla^{2}$ mean that, if $\Delta^{2} \propto k^{4}$ for the power spectrum of density fluctuations, then $\Delta_{\Phi}^{2}$ is a constant. Since potential perturbations govern the flatness of spacetime, this says that the scaleinvariant spectrum corresponds to a metric that is a fractal: spacetime has the same degree of 'wrinkliness' on each resolution scale. The total curvature fluctuations diverge, but only logarithmically at either extreme of wavelength.

\subsection{ERROR ESTIMATES}

A key question for these statistical measures is how accurate they are i.e. how much does the result for a given finite sample depart from the ideal statistic averaged over an infinite universe? Terminology here can be confusing, in that a distinction is sometimes made between sampling variance and cosmic variance. The former is to be understood as arising from probing a given volume only with a finite number of galaxies (e.g. just the bright ones), so that $\sqrt{N}$ statistics limit our knowledge of the mass distribution within that region. The second term concerns whether we have reached a fair sample of the universe, and depends on whether there is significant power in density perturbation modes with wavelengths larger than the sample depth. Clearly, these two aspects are closely related.

The quantitative analysis of these errors is most simply performed in Fourier space, and was given by Feldman, Kaiser \& Peacock (1994). The results can be understood most simply by comparison with an idealized complete and uniform survey of a volume $L^{3}$, with periodicity scale $L$. For an infinite survey, the arbitrariness of the spatial origin means that different modes are uncorrelated:

$$
\left\langle\delta_{k}\left(\mathbf{k}_{i}\right) \delta_{k}^{*}\left(\mathbf{k}_{j}\right)\right\rangle=P(k) \delta_{i j} .
$$


Each mode has an exponential distribution in power (because the complex coefficients $\delta_{k}$ are 2D Gaussian-distributed variables on the Argand plane), for which the mean and rms are identical. The fractional uncertainty in the mean power measured over some $k$-space volume is then just determined by the number of uncorrelated modes averaged over:

$$
\frac{\delta \bar{P}}{\bar{P}}=\frac{1}{N_{\text {modes }}^{1 / 2}} ; \quad N_{\text {modes }}=\left(\frac{L}{2 \pi}\right)^{3} \int d^{3} k .
$$

The only subtlety is that, because the density field is real, modes at $k$ and $-k$ are perfectly correlated. Thus, if the $k$-space volume is a shell, the effective number of uncorrelated modes is only half the above expression.

Analogous results apply for an arbitrary survey selection function. In the continuum limit, the Kroneker delta in the expression for mode correlation would be replaced a term proportional to a delta-function, $\left.\delta\left[\mathbf{k}_{i}-\mathbf{k}_{j}\right]\right)$. Now, multiplying the infinite ideal survey by a survey window, $\rho(\mathbf{r})$, is equivalent to convolution in the Fourier domain, with the result that the power per mode is correlated over $k$-space separations of order $1 / D$, where $D$ is the survey depth.

Given this expression for the fractional power, it is clear that the precision of the estimate can be manipulated by appropriate weighting of the data: giving increased weight to the most distant galaxies increases the effective survey volume, boosting the number of modes. This sounds too good to be true, and of course it is: the above expression for the fractional power error applies to the sum of true clustering power and shot noise. The latter arises because we transform a point process. Given a set of $N$ galaxies, we would estimate Fourier coefficients via $\delta_{k}=(1 / N) \sum_{i} \exp \left(-i \mathbf{k} \cdot x_{i}\right)$. From this, the expectation power is

$$
\left\langle\left|\delta_{k}\right|^{2}\right\rangle=P(k)+1 / N
$$

The existence of an additive discreteness correction is no problem, but the fluctuations on the shot noise hide the signal of interest. Introducing weights boosts the shot noise, so there is an optimum choice of weight that minimizes the uncertainty in the power after shot-noise subtraction. Feldman, Kaiser \& Peacock (1994) showed that this weight is

$$
w=(1+\bar{n} P)^{-1},
$$

where $\bar{n}$ is the expected galaxy number density as a function of position in the survey.

Since the correlation of modes arises from the survey selection function, it is clear that weighting the data changes the degree of correlation in $k$ 
space. Increasing the weight in low-density regions increases the effective survey volume, and so shrinks the $k$-space coherence scale. However, the coherence scale continues to shrink as distant regions of the survey are given greater weight, whereas the noise goes through a minimum. There is thus a trade-off between the competing desirable criteria of high $k$-space resolution and low noise. Tegmark (1996) shows how weights may be chosen to implement any given prejudice concerning the relative importance of these two criteria. See also Hamilton $(1997 b, c)$ for similar arguments.

\subsection{KARHUNEN-LOÈVE AND ALL THAT}

Given these difficulties with correlated results, it is attractive to seek a method where the data can be decomposed into a set of statistics that are completely uncorrelated with each other. Such a method is provided by the Karhunen-Loève formalism. Vogeley \& Szalay (1996) argued as follows. Define a column vector of data $\underline{d}$; this can be quite abstract in nature, and could be e.g. the numbers of galaxies in a set of cells, or a set of Fourier components of the transformed galaxy number counts. Similarly, for CMB studies, $\underline{d}$ could be $\delta T / T$ in a set of pixels, or spherical-harmonic coefficients $a_{\ell m}$. We assume that the mean can be identified and subtracted off, so that $\langle\underline{d}\rangle=0$ in ensemble average. The statistical properties of the data are then described by the covariance matrix

$$
C_{i j} \equiv\left\langle d_{i} d_{j}^{*}\right\rangle
$$

(normally the data will be real, but it is convenient to keep things general and include the complex conjugate).

Suppose we seek to expand the datavector in terms of a set of new orthonormal vectors:

$$
\underline{d}=\sum_{i} a_{i} \underline{\psi}_{i} ; \quad \underline{\psi}_{i}^{*} \cdot \underline{\psi}_{j}=\delta_{i j}
$$

The expansion coefficients are extracted in the usual way: $a_{j}=\underline{d} \cdot \underline{\psi}_{j}^{*}$. Now require that these coefficients be statistically uncorrelated, $\left\langle a_{i} a_{j}^{*}\right\rangle=\lambda_{i} \delta_{i j}$ (no sum on $i$ ). This gives

$$
\underline{\psi}_{i}^{*} \cdot\left\langle\underline{d} \underline{d}^{*}\right\rangle \cdot \underline{\psi}_{j}=\lambda_{i} \delta_{i j},
$$

where the dyadic $\left\langle\underline{d} \underline{d}^{*}\right\rangle$ is $\underline{\underline{C}}$, the correlation matrix of the data vector: $\left(\underline{d} \underline{d}^{*}\right)_{i j} \equiv d_{i} d_{j}^{*}$. Now, the effect of operating this matrix on one of the $\underline{\psi}_{i}$ must be expandable in terms of the complete set, which shows that the $\underline{\psi}_{j}$ must be the eigenvectors of the correlation matrix:

$$
\left\langle\underline{d} \underline{d}^{*}\right\rangle \cdot \underline{\psi}_{j}=\lambda_{j} \underline{\psi}_{j}
$$


Vogeley \& Szalay further show that these uncorrelated modes are optimal for representing the data: if the modes are arranged in order of decreasing $\lambda$, and the series expansion truncated after $n$ terms, the rms truncation error is minimized for this choice of eigenmodes. To prove this, consider the truncation error

$$
\underline{\epsilon}=\underline{d}-\sum_{i=1}^{n} a_{i} \underline{\psi}_{i}=\sum_{i=n+1}^{\infty} a_{i} \underline{\psi}_{i}
$$

The square of this is

$$
\left\langle\epsilon^{2}\right\rangle=\sum_{i=n+1}^{\infty}\left\langle\left|a_{i}\right|^{2}\right\rangle,
$$

where $\left\langle\left|a_{i}\right|^{2}\right\rangle=\underline{\psi}_{i}^{*} \cdot \underline{\underline{C}} \cdot \underline{\psi}_{i}$, as before. We want to minimize $\left\langle\epsilon^{2}\right\rangle$ by varying the $\psi_{i}$, but we need to do this in a way that preserves normalization. This is achieved by introducing a Lagrange multiplier, and minimizing

$$
\sum \underline{\psi}_{i}^{*} \cdot \underline{\underline{C}} \cdot \underline{\psi}_{i}+\lambda\left(1-\underline{\psi}_{i}^{*} \cdot \underline{\psi}_{i}\right)
$$

This is easily solved if we consider the more general problem where $\underline{\psi}_{i}^{*}$ and $\underline{\psi}_{i}$ are independent vectors:

$$
\underline{\underline{C}} \cdot \underline{\psi}_{i}=\lambda \psi_{i}
$$

In short, the eigenvectors of $\underline{\underline{C}}$ are optimal in a least-squares sense for expanding the data. The process of truncating the expansion is a form of lossy data compression, since the size of the data vector can be greatly reduced without significantly affecting the fidelity of the resulting representation of the universe.

The process of diagonalizing the covariance matrix of a set of data also goes by the more familiar name of principal components analysis, so what is the difference between the KL approach and PCA? In the above discussion, they are identical, but the idea of choosing an optimal eigenbasis is more general than PCA. Consider the case where the covariance matrix can be decomposed into a 'signal' and a 'noise' term:

$$
\underline{\underline{C}}=\underline{\underline{S}}+\underline{\underline{N}}
$$

where $\underline{\underline{S}}$ depends on cosmological parameters that we might wish to estimate, whereas $\underline{\underline{N}}$ is some fixed property of the experiment under consideration. In the simplest imaginable case, $\underline{\underline{N}}$ might be a diagonal matrix, so PCA diagonalizes both $\underline{\underline{S}}$ and $\underline{\underline{N}}$. In this case, ranking the PCA modes by 
eigenvalue would correspond to ordering the modes according to signal-tonoise ratio. Data compression by truncating the mode expansion then does the sensible thing: it rejects all modes of low signal-to-noise ratio.

However, in general these matrices will not commute, and there will not be a single set of eigenfunctions that are common to the $\underline{\underline{S}}$ and $\underline{\underline{N}}$ matrices. Normally, this would be taken to mean that it is impossible to find a set of coordinates in which both are diagonal. This conclusion can however be evaded, as follows. When considering the effect of coordinate transformations on vectors and matrices, we are normally forced to consider only rotation-like transformations that preserve the norm of a vector (e.g. in quantum mechanics, so that states stay normalized). Thus, we write $\underline{d}^{\prime}=$ $\underline{\underline{R}} \cdot \underline{d}$, where $\underline{\underline{R}}$ is unitary, so that $\underline{\underline{R}} \cdot \underline{\underline{R}}^{\dagger}=\underline{\underline{I}}$. If $\underline{\underline{R}}$ is chosen so that its columns are the eigenvalues of $\underline{\underline{N}}$, then the transformed noise matrix, $\underline{\underline{R}} \cdot \underline{\underline{N}} \cdot \underline{\underline{R}}^{\dagger}$, is diagonal. Nevertheless, if the transformed $\underline{\underline{S}}$ is not diagonal, the two will not commute. This apparently insuperable problem can be solved by using the fact that the data vectors are entirely abstract at this stage. There is therefore no reason not to consider the further transformation of scaling the data, so that $\underline{\underline{N}}$ becomes proportional to the identity matrix. This means that the transformation is no longer unitary - but there is no physical reason to object to a change in the normalization of the data vectors.

Suppose we therefore make a further transformation

$$
\underline{d}^{\prime \prime}=\underline{\underline{W}} \cdot \underline{d}^{\prime}
$$

The matrix $\underline{\underline{W}}$ is related to the rotated noise matrix:

$$
\underline{\underline{N}}^{\prime}=\operatorname{diag}\left(n_{1}, n_{2}, \ldots\right) \quad \Rightarrow \quad \underline{\underline{W}}=\operatorname{diag}\left(1 / \sqrt{n_{1}}, 1 / \sqrt{n_{2}}, \ldots\right) .
$$

This transformation is termed prewhitening by Vogeley \& Szalay (1996), since it converts the noise matrix to white noise, in which each pixel has a unit noise that is uncorrelated with other pixels. The effect of this transformation on the full covariance matrix is

$$
C_{i j}^{\prime \prime} \equiv\left\langle d_{i}^{\prime \prime} d_{j}^{\prime *}\right\rangle \quad \Rightarrow \quad \underline{\underline{C}}^{\prime \prime}=(\underline{\underline{W}} \cdot \underline{\underline{R}}) \cdot \underline{\underline{C}} \cdot(\underline{\underline{W}} \cdot \underline{\underline{R}})^{\dagger}
$$

After this transformation, the noise and signal matrices certainly do commute, and the optimal modes for expanding the new data are once again the PCA eigenmodes in the new coordinates:

$$
\underline{\underline{C}}^{\prime \prime} \cdot \underline{\psi}_{i}^{\prime \prime}=\lambda \underline{\psi}_{i}^{\prime \prime}
$$

These eigenmodes must be expressible in terms of some modes in the original coordinates, $\underline{e}_{i}$ :

$$
\underline{\psi}_{i}^{\prime \prime}=(\underline{\underline{W}} \cdot \underline{\underline{R}}) \cdot \underline{e}_{i} .
$$


In these terms, the eigenproblem is

$$
(\underline{\underline{W}} \cdot \underline{\underline{R}}) \cdot \underline{\underline{C}} \cdot(\underline{\underline{W}} \cdot \underline{\underline{R}})^{\dagger} \cdot(\underline{\underline{W}} \cdot \underline{\underline{R}}) \cdot \underline{e}_{i}=\lambda(\underline{\underline{W}} \cdot \underline{\underline{R}}) \cdot \underline{e}_{i}
$$

This can be simplified using $\underline{\underline{W}}^{\dagger} \cdot \underline{\underline{W}}=\underline{\underline{N}}^{\prime-1}$ and $\underline{\underline{N}}^{\prime-1}=\underline{\underline{R}} \cdot \underline{\underline{N}}^{-1} \underline{\underline{R}}{ }^{\dagger}$, to give

$$
\underline{\underline{C}} \cdot \underline{\underline{N}}^{-1} \cdot \underline{e}_{i}=\lambda \underline{e}_{i}
$$

so the required modes are eigenmodes of $\underline{\underline{C}} \cdot \underline{\underline{N}}^{-1}$. However, care is required when considering the orthonormality of the $\underline{e}_{i}: \underline{\psi}_{i}^{\dagger} \cdot \underline{\psi}_{j}=\underline{e}_{i}^{\dagger} \cdot \underline{\underline{N}}^{-1} \cdot \underline{e}_{j}$, so the $\underline{e}_{i}$ are not orthonormal. If we write $\underline{d}=\sum_{i} a_{i} \underline{e}_{i}$, then

$$
a_{i}=\left(\underline{\underline{N}}^{-1} \cdot \underline{e}_{i}\right)^{\dagger} \cdot \underline{d} \equiv \underline{\psi}_{i}^{\dagger} \cdot \underline{d} .
$$

Thus, the modes used to extract the compressed data by dot product satisfy $\underline{\underline{C}} \cdot \underline{\psi}=\lambda \underline{\underline{N}} \cdot \underline{\psi}$, or finally

$$
\underline{\underline{S}} \cdot \underline{\psi}=\lambda \underline{\underline{N}} \cdot \underline{\psi}
$$

given a redefinition of $\lambda$. The optimal modes are thus eigenmodes of $\underline{N}^{-1} \cdot \underline{\underline{S}}$, hence the name signal-to-noise eigenmodes (Bond 1995; Bunn 1996).

It is interesting to appreciate that the set of KL modes just discussed is also the 'best' set of modes to choose from a completely different point of view: they are the modes that are optimal for estimation of a parameter via maximum likelihood. Suppose we write the compressed data vector, $\underline{x}$, in terms of a non-square matrix $\underline{\underline{A}}$ (whose rows are the basis vectors $\underline{\psi}_{i}^{*}$ ):

$$
\underline{x}=\underline{A} \cdot \underline{d} .
$$

The transformed covariance matrix is

$$
\underline{\underline{D}} \equiv\left\langle\underline{x} \underline{x}^{\dagger}\right\rangle=\underline{\underline{A}} \cdot \underline{\underline{C}} \cdot \underline{\underline{A}}^{\dagger} .
$$

For the case where the original data obeyed Gaussian statistics, this is true for the compressed data also, so the likelihood is

$$
-2 \ln \mathcal{L}=\ln \operatorname{det} \underline{\underline{D}}+\underline{x}^{*} \cdot \underline{\underline{D}}^{-1} \cdot \underline{x}+\mathrm{constant}
$$

The normal variance on some parameter $p$ (on which the covariance matrix depends) is

$$
\frac{1}{\sigma_{p}^{2}}=\frac{d^{2}[-2 \ln \mathcal{L}]}{d q^{2}}
$$


Without data, we don't know this, so it is common to use the expectation value of the rhs as an estimate (recently, there has been a tendency to dub this the 'Fisher matrix').

We desire to optimize $\sigma_{p}$ by an appropriate choice of data-compression vectors, $\underline{\psi}_{i}$. By writing $\sigma_{p}$ in terms of $\underline{\underline{A}}, \underline{\underline{C}}$ and $\underline{d}$, it may eventually be shown that the desired optimal modes satisfy

$$
\left(\frac{d}{d p} \underline{\underline{C}}\right) \cdot \underline{\psi}=\lambda \underline{\underline{C}} \cdot \underline{\psi}
$$

For the case where the parameter of interest is the cosmological power, the matrix on the lhs is just proportional to $\underline{\underline{S}}$, so we have to solve the eigenproblem

$$
\underline{\underline{S}} \cdot \underline{\psi}=\lambda \underline{\underline{C}} \cdot \underline{\psi} .
$$

With a redefinition of $\lambda$, this becomes

$$
\underline{\underline{S}} \cdot \underline{\psi}=\lambda \underline{\underline{N}} \cdot \underline{\psi} .
$$

The optimal modes for parameter estimation in the linear case are thus identical to the PCA modes of the prewhitened data discussed above. The more general expression was given by Tegmark, Taylor \& Heavens (1997), and it is only in this case, where the covariance matrix is not necessarily linear in the parameter of interest, that the KL method actually differs from PCA.

The reason for going to all this trouble is that the likelihood can now be evaluated much more rapidly, using the compressed data. This allows extensive model searches over large parameter spaces that would be unfeasible with the original data (since inversion of an $N \times N$ covariance matrix takes a time proportional to $N^{3}$ ). Note however that the price paid for this efficiency is that a different set of modes need to be chosen depending on the model of interest, and that these modes will not in general be optimal for expanding the dataset itself. Nevertheless, it may be expected that application of these methods will inevitably grow as datasets increase in size. Present applications mainly prove that the techniques work: see Matsubara, Szalay \& Landy (1999) for application to the LCRS, or Padmanabhan, Tegmark \& Hamilton (1999) for the UZC survey. The next generation of experiments will probably be forced to resort to data compression of this sort, rather than using it as an elegant alternative method of analysis.

\section{Redshift-space effects}

Peculiar velocity fields are responsible for the distortion of the clustering pattern in redshift space, as first clearly articulated by Kaiser (1987). For 
a survey that subtends a small angle (i.e. in the distant-observer approximation), a good approximation to the anisotropic redshift-space Fourier spectrum is given by the Kaiser function together with a damping term from nonlinear effects:

$$
\delta_{k}^{s}=\delta_{k}^{r}\left(1+\beta \mu^{2}\right) D(k \sigma \mu),
$$

where $\beta=\Omega_{m}^{0.6} / b, b$ being the linear bias parameter of the galaxies under study, and $\mu=\hat{\mathbf{k}} \cdot \hat{\mathbf{r}}$. For an exponential distribution of relative small-scale peculiar velocities (as seen empirically), the damping function is $D(y) \simeq$

$\left(1+y^{2} / 2\right)^{-1 / 2}$, and $\sigma \simeq 400 \mathrm{~km} \mathrm{~s}^{-1}$ is a reasonable estimate for the pairwise velocity dispersion of galaxies (e.g. Ballinger, Peacock \& Heavens 1996).

In principle, this distortion should be a robust way to determine $\Omega$ (or at least $\beta$ ). In practice, the effect has not been easy to see with past datasets. This is mainly a question of depth: a large survey is needed in order to beat down the shot noise, but this tends to favour bright spectroscopic limits. This limits the result both because relatively few modes in the linear regime are sampled, and also because local survey volumes will tend to violate the small-angle approximation. Strauss \& Willick (1995) and Hamilton (1997a) review the practical application of redshift-space distortions. In the next section, preliminary results are presented from the $2 \mathrm{dF}$ redshift survey, which shows the distortion effect clearly for the first time.

\section{The state of the art in LSS}

\subsection{THE APM SURVEY}

In the past few years, much attention has been attracted by the estimate of the galaxy power spectrum from the APM survey (Baugh \& Efstathiou 1993, 1994; Maddox et al. 1996). The APM result was generated from a catalogue of $\sim 10^{6}$ galaxies derived from UK Schmidt Telescope photographic plates scanned with the Cambridge Automatic Plate Measuring machine; because it is based on a deprojection of angular clustering, it is immune to the complicating effects of redshift-space distortions. The difficulty, of course, is in ensuring that any low-level systematics from e.g. spatial variations in magnitude zero point are sufficiently well controlled that they do not mask the cosmological signal, which is of order $w(\theta) \lesssim 0.01$ at separations of a few degrees.

The best evidence that the APM survey has the desired uniformity is the scaling test, where the correlations in fainter magnitude slices are expected to move to smaller scales and be reduced in amplitude. If we increase the depth of the survey by some factor $D$, the new angular correlation function 
will be

$$
w^{\prime}(\theta)=\frac{1}{D} w(D \theta)
$$

The APM survey passes this test well; once the overall redshift distribution is known, it is possible to obtain the spatial power spectrum by inverting a convolution integral:

$$
w(\theta)=\int_{0}^{\infty} y^{4} \phi^{2} d y \int_{0}^{\infty} \pi \Delta^{2}(k) J_{0}(k y \theta) d k / k^{2}
$$

(where zero spatial curvature is assumed). Here, $\phi(y)$ is the comoving density at comoving distance $y$, normalized so that $\int y^{2} \phi(y) d y=1$.

This integral was inverted numerically by Baugh \& Efstathiou (1993), and gives an impressively accurate determination of the power spectrum. The error estimates are derived empirically from the scatter between independent regions of the sky, and so should be realistic. If there are no undetected systematics, these error bars say that the power is very accurately determined. The APM result has been investigated in detail by a number of authors (e.g. Gaztañaga \& Baugh 1998; Eisenstein \& Zaldarriaga 1999) and found to be robust; this has significant implications if true.

\subsection{PAST REDSHIFT SURVEYS}

Because of the sheer number of galaxies, plus the large volume surveyed, the APM survey outperforms redshift surveys of the past, at least for the purpose of determining the power spectrum. The largest surveys of recent years (CfA: Huchra et al. 1990; LCRS: Shectman et al. 1996; PSCz: Saunders et al. 1999) contain of order $10^{4}$ galaxy redshifts, and their statistical errors are considerably larger than those of the APM. On the other hand, it is of great importance to compare the results of deprojection with clustering measured directly in $3 \mathrm{D}$.

This comparison was carried out by Peacock \& Dodds (1994; PD94). The exercise is not straightforward, because the $3 \mathrm{D}$ results are affected by redshift-space distortions; also, different galaxy tracers can be biased to different extents. The approach taken was to use each dataset to reconstruct an estimate of the linear spectrum, allowing the relative bias factors to float in order to make these estimates agree as well as possible (figure 5). To within a scatter of perhaps a factor 1.5 in power, the results were consistent with a $\Gamma \simeq 0.25 \mathrm{CDM}$ model. Even though the subsequent sections will discuss some possible disagreements with the CDM models at a higher level of precision, the general existence of CDM-like curvature in the spectrum is likely to be an important clue to the nature of the dark matter. 

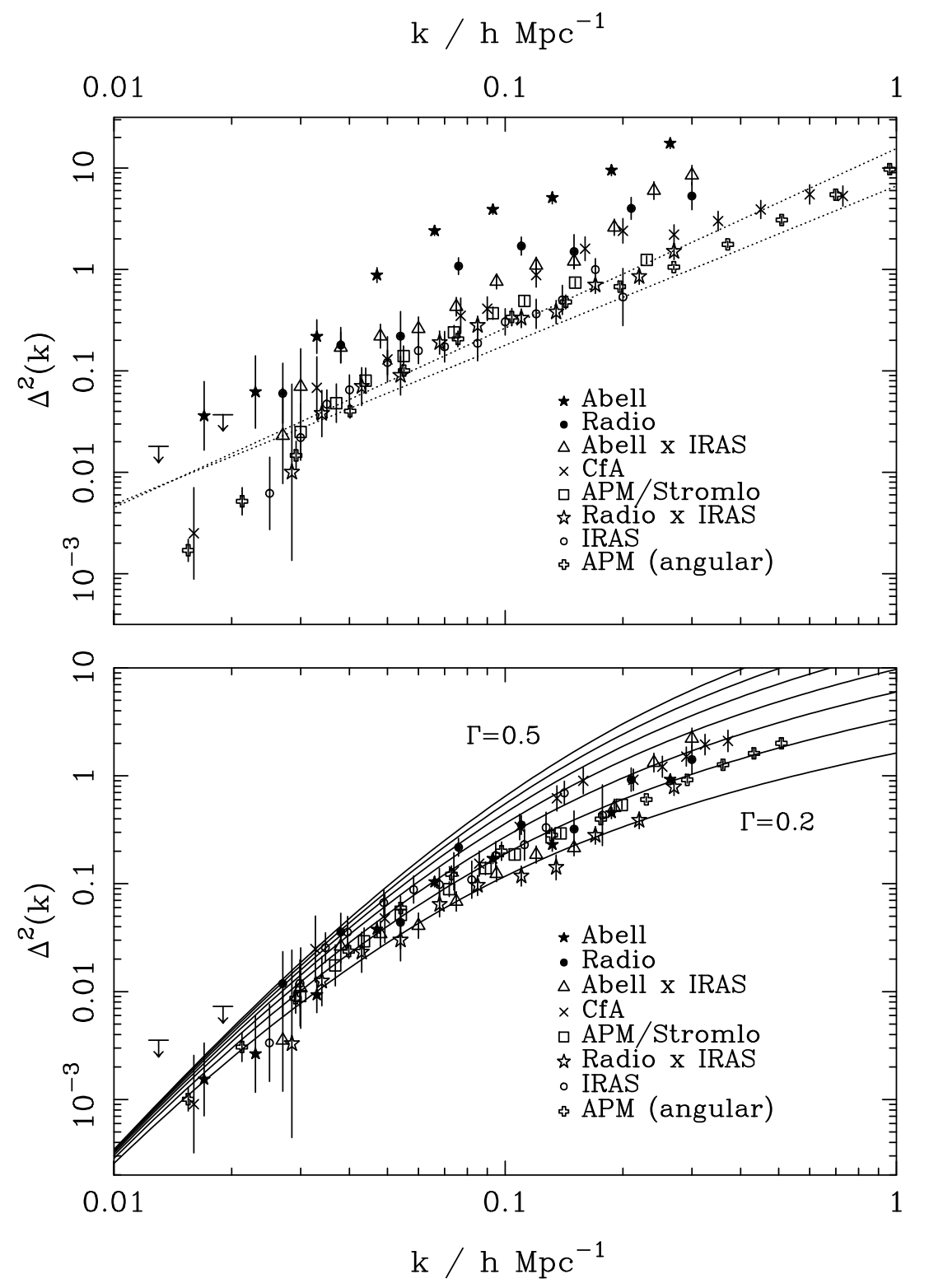

Figure 5. The PD94 compilation of power-spectrum measurements. The upper panel shows raw power measurements; the lower shows these data corrected for relative bias, nonlinear effects, and redshift-space effects.

\subsection{THE 2DF SURVEY}

The proper resolution of many of the observational questions regarding the large-scale distribution of galaxies requires new generations of redshift 


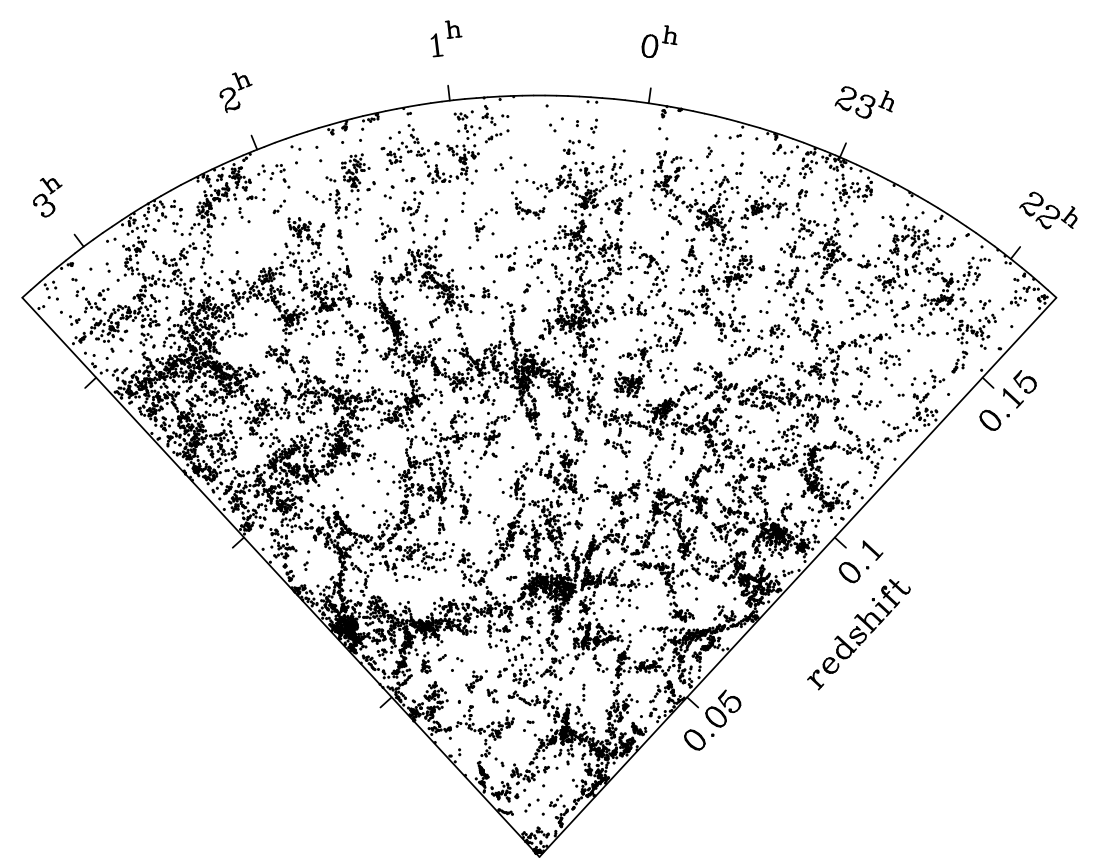

Figure 6. A 4-degree thick slice of the Southern strip of the 2 dF redshift survey. This restricted region alone contains 16,419 galaxies.

survey that push beyond the $N=10^{5}$ barrier. Two groups are pursuing this goal. The Sloan survey (e.g. Margon 1999) is using a dedicated 2.5-m telescope to measure redshifts for approximately 700,000 galaxies to $r=18.2$ in the North Galactic Cap. The 2dF survey (e.g. Colless 1999) is using a fraction of the time on the 3.9-m Anglo-Australian Telescope plus Two-Degree Field spectrograph to measure 250,000 galaxies from the APM survey to $B_{J}=19.45$ in the South Galactic Cap. At the time of writing, the Sloan spectroscopic survey has yet to commence. However, the $2 \mathrm{dF}$ project has measured 77,000 redshifts, and some preliminary clustering results are given below. For more details of the survey, particularly the team members whose hard work has made all this possible, see http://www.mso.anu.edu.au/2dFGRS/.

One of the advantages of $2 \mathrm{dF}$ is that it is a fully sampled survey, so that the space density out to the depth imposed by the magnitude limit (median $z=0.12$ ) is as high as nature allows: apart from a tail of low surface brightness galaxies (inevitably omitted from any spectroscopic survey), the $2 \mathrm{dF}$ measure all the galaxies that exist over a cosmologically representative volume. It is the first to achieve this goal. The fidelity of the resulting map 
of the galaxy distribution can be seen in figure 6 , which shows a small subset of the data: a slice of thickness 4 degrees, centred at declination $-27^{\circ}$.

An issue with using the $2 \mathrm{dF}$ data in their current form is that the sky has to be divided into circular 'tiles' each two degrees in diameter (' $2 \mathrm{dF}$ ' $=$ 'two-degree field', within which the AAT is able to measure 400 spectra simultaneously; see http://www . aao.gov . au/2df/for details of the instrument). The tiles are positioned adaptively, so that larger overlaps occur in regions of high galaxy density. It this way, it is possible to place a fibre on $>95 \%$ of all galaxies. However, while the survey is in progress, there exist parts of the sky where the overlapping tiles have not yet been observed, and so the effective sampling fraction is only $\simeq 50 \%$. These effects can be allowed for in two different ways. In clustering analyses, we compare the counts of pairs (or $n$-tuplets) of galaxies in the data to the corresponding counts involving an unclustered random catalogue. The effects of variable sampling can therefore be dealt with either by making the density of random points fluctuate according to the sampling, or by weighting observed galaxies by the reciprocal of the sampling factor for the zone in which they lie. The former approach is better from the point of view of shot noise, but the latter may be safer if there is any suspicion that the sampling fluctuations are correlated with real structure on the sky. In practice, both strategies give identical answers for the results below.

At the two-point level, the most direct quantity to compute is the redshift-space correlation function. This is an anisotropic function of the orientation of a galaxy pair, owing to peculiar velocities. We therefore evaluate $\xi$ as a function of $2 \mathrm{D}$ separation in terms of coordinates both parallel and perpendicular to the line of sight. If the comoving radii of two galaxies are $y_{1}$ and $y_{2}$ and their total separation is $r$, then we define coordinates

$$
\pi \equiv\left|y_{1}-y_{2}\right| ; \quad \sigma=\sqrt{r^{2}-\pi^{2}}
$$

The correlation function measured in these coordinates is shown in figure 7. In evaluating $\xi(\sigma, \pi)$, the optimal radial weight discussed above has been applied, so that the noise at large $r$ should be representative of true cosmic scatter.

The correlation-function results display very clearly the two signatures of redshift-space distortions discussed above. The fingers of God from smallscale random velocities are very clear, as indeed has been the case from the first redshift surveys (e.g. Davis \& Peebles 1983). However, this is arguably the first time that the large-scale flattening from coherent infall has been really obvious in the data.

A good way to quantify the flattening is to analyze the clustering as a 

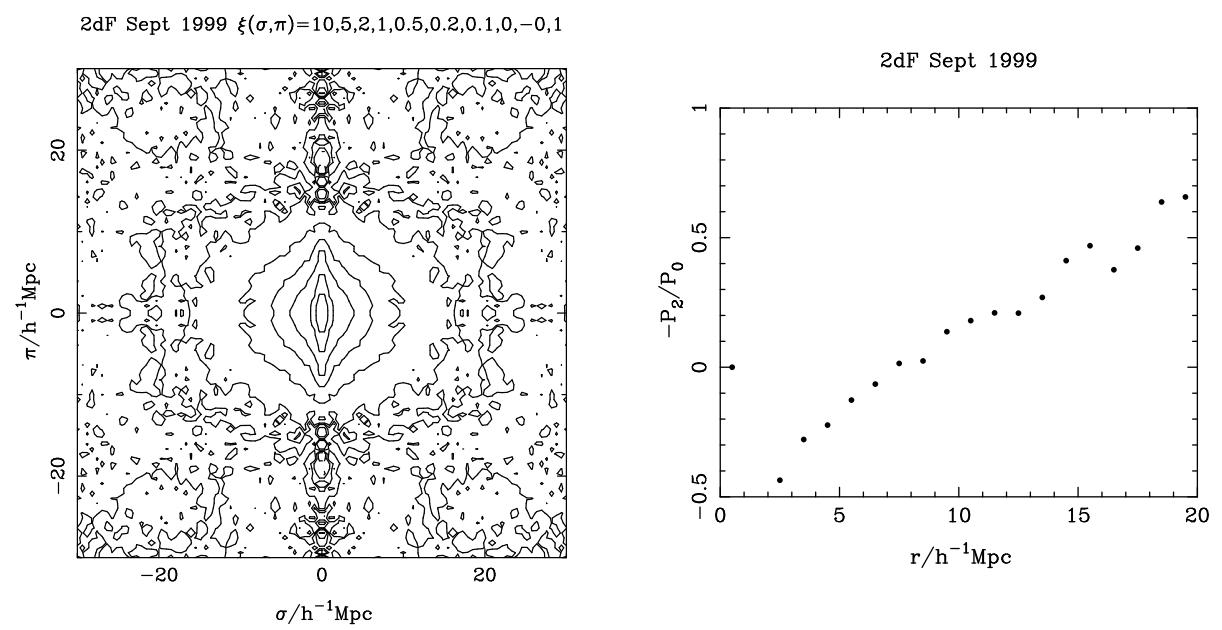

Figure 7. The redshift-space correlation function from the $2 \mathrm{dF}$ data, $\xi(\sigma, \pi)$, with a bin size of $0.6 h^{-1}$ Mpc. $\sigma$ is the pair separation transverse to the line of sight; $\pi$ is the radial separation. This plot clearly displays redshift distortions, with 'fingers of God' at small scales and the coherent Kaiser squashing at large $\sigma$. The distortions are quantified via the quadrupole-to-monopole ratio of $\xi$ as a function of radius in the second panel. The contours are round at $r=7 h^{-1} \mathrm{Mpc}$, but flatten progressively thereafter.

function of angle into Legendre polynomials:

$$
\xi_{\ell}(r)=\frac{2 \ell+1}{2} \int_{-1}^{1} \xi(\sigma=r \sin \theta, \pi=r \cos \theta) P_{\ell}(\cos \theta) d \cos \theta .
$$

The quadrupole-to-monopole ratio should be a clear indicator of coherent infall. In linear theory, it is given by

$$
\frac{\xi_{2}}{\xi_{0}}=f(n) \frac{4 \beta / 3+4 \beta^{2} / 7}{1+2 \beta / 3+\beta^{2} / 5}
$$

where $f(n)=(3+n) / n$ (Hamilton 1992). On small and intermediate scales, the effective spectral index is negative, so the quadrupole-to-monopole ratio should be negative, as observed.

However, it is clear that the results on the largest scales are still significantly affected by finger-of-God smearing. The best way to interpret the observed effects is to calculate the same quantities for a model. To achieve this, we use the observed APM 3D power spectrum, plus the distortion model discussed above. This gives the plots shown in figure 8. The free parameter is $\beta$, and this is set at a value of 0.5 , approximately consistent with other arguments for a universe with $\Omega=0.3$ and little large-scale bias (e.g. Peacock 1997). Although a quantitative comparison has not yet been carried out, it is clear that this plot closely resembles the observed data. 

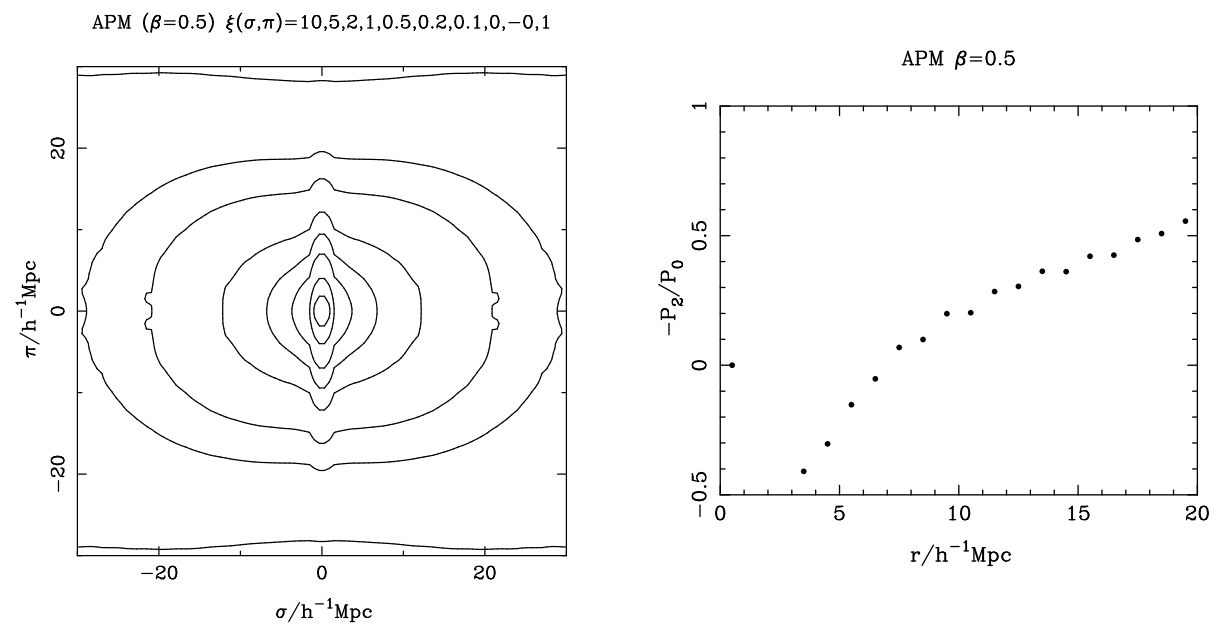

Figure 8. The redshift-space correlation function predicted from the real-space APM power spectrum, assuming the model of Ballinger, Peacock \& Heavens (1996), with $\beta=0.5$.

By the end of 2001, the size of the $2 \mathrm{dF}$ survey should have expanded by a factor 3 , increasing the pair counts tenfold. It should then be possible to trace the correlations well beyond the present limit, and follow the redshift-space distortion well into the linear regime. However, the biggest advantage of a survey of this size and uniformity is the ability to subdivide it. All analyses to date have lumped together very different kinds of galaxies, whereas we know from morphological segregation that different classes of galaxy have spatial distributions that differ from each other. The homogeneous $2 \mathrm{dF}$ data allow classification into different galaxy types (representing, physically, a sequence of star-formation rates), from the spectra alone (Folkes et al. 1999). It will be a critical test to see if the distortion signature can be picked up in each type individually. Although the large-scale behaviour of each galaxy type will probably be quite similar, differences in the clustering properties will inevitably arise on smaller scales, giving important information about the sequence of galaxy formation.

\section{Small-scale clustering}

\subsection{HISTORY}

One of the earliest models to be used to interpret the galaxy correlation function was to consider a density field composed of randomly-placed independent clumps with some universal density profile (Neyman, Scott \& Shane 1953; Peebles 1974). Since the clumps are placed at random, the 
only correlations arise from points in the same clump. The correlations are easily deduced by using statistical isotropy: calculate the excess number of pairs separated by a distance $r$ in the $z$ direction (chosen as some arbitrary polar axis in a spherically-symmetric clump). For power-law clumps, with $\rho=n B r^{-\epsilon}$, truncated at $r=R$, this model gives $\xi \propto r^{3-2 \epsilon}$ in the limit $r \ll R$, provided $3 / 2<\epsilon<3$. Values $\epsilon>3$ are unphysical, and require a small-scale cutoff to the profile. There is no such objection to $\epsilon<3 / 2$, and the expression for $\xi$ tends to a constant for small $r$ in this case (see Yano \& Gouda 1999).

A long-standing problem for this model is that the correlation function in this case is much flatter than is observed for galaxies: $\xi \propto r^{-1.8}$ is the canonical slope, requiring $\epsilon=2.4$. The first reaction may be to say that the model is incredibly naive by comparison with our sophisticated present understanding of the nonlinear evolution of CDM density fields. However, as will be shown below, it may after all contain more than a grain of truth.

\subsection{THE CDM CLUSTERING PROBLEMS}

A number of authors have pointed out that the detailed spectral shape inferred from galaxy data appears to be inconsistent with that of nonlinear evolution from CDM initial conditions. (e.g. Efstathiou, Sutherland \& Maddox 1990; Klypin, Primack \& Holtzman 1996; Peacock 1997). Perhaps the most detailed work was carried out by the VIRGO consortium, who carried out $N=256^{3}$ simulations of a number of CDM models (Jenkins et al. 1998). Their results are shown in figure 9, which gives the nonlinear power spectrum at various times (cluster normalization is chosen for $z=0$ ) and contrasts this with the APM data. The lower small panels are the scale-dependent bias that would required if the model did in fact describe the real universe, defined as

$$
b(k) \equiv\left(\frac{\Delta_{\text {gals }}^{2}(k)}{\Delta_{\text {mass }}^{2}}\right)^{1 / 2}
$$

In all cases, the required bias is non-monotonic; it rises at $k \gtrsim 5 h^{-1} \mathrm{Mpc}$, but also displays a bump around $k \simeq 0.1 h^{-1} \mathrm{Mpc}$. If real, this feature seems impossible to understand as a genuine feature of the mass power spectrum; certainly, it is not at a scale where the effects of even a large baryon fraction would be expected to act (Eisenstein et al. 1998; Meiksin, White \& Peacock 1999). 

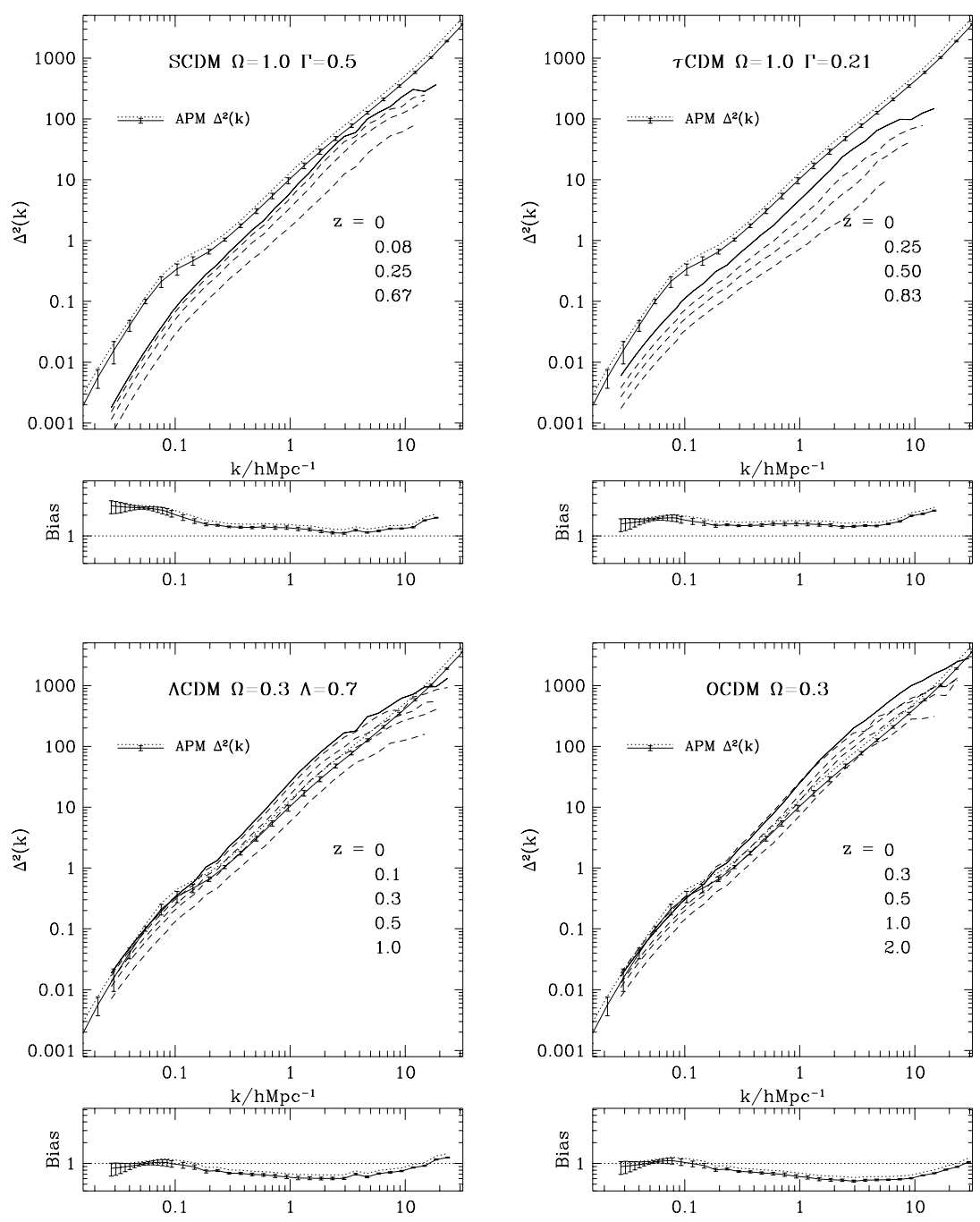

Figure 9. The nonlinear evolution of various CDM power spectra, as determined by the Virgo consortium (Jenkins et al. 1998).

\section{Bias}

The conclusions from the above discussion are either that the physics of dark matter and structure formation are more complex than in CDM models, or that the relation between galaxies and the overall matter distribution is sufficiently complicated that the effective bias is not a simple slowlyvarying monotonic function of position. 


\subsection{SIMPLE BIAS MODELS}

The simplest assumption is that all the complicated physical effects leading to galaxy formation depend in a causal (but nonlinear) way on the local mass density, so that we write

$$
\rho_{\text {light }}=f\left(\rho_{\text {mass }}\right) .
$$

Coles (1993) showed that, under rather general assumptions, this equation would lead to an effective bias that was a monotonic function of scale. This issue was investigated in some detail by Mann, Peacock \& Heavens (1998), who verified Coles' conclusion in practice for simple few-parameter forms for $f$, and found in all cases that the effective bias varied rather weakly with scale. The APM results thus are either inconsistent with a CDM universe, or require non-local bias.

A puzzle with regard to this conclusion is provided by the work of Jing, Mo \& Börner (1998). They evaluated the projected real-space correlations for the LCRS survey (see figure 10). This statistic also fails to match the prediction of CDM models, but this can be amended by introducing a simple antibias scheme, in which galaxy formation is suppressed in the most massive haloes. This scheme should in practice be very similar to the Mann, Peacock \& Heavens recipe of a simple weighting of particles as a function of the local density; indeed, the main effect is a change of amplitude, rather than shape of the correlations. The puzzle is this: if the APM power spectrum is used to predict the projected correlation function, the result agrees almost exactly with the LCRS. Either projected correlations are a rather insensitive statistic, or perhaps the Baugh \& Efstathiou deconvolution procedure used to get $P(k)$ has exaggerated the significance of features in the spectrum. The LCRS results are one reason for treating the apparent conflict between APM and CDM with caution.

\subsection{HALO CORRELATIONS}

In reality, bias is unlikely to be completely causal, and this has led some workers to explore stochastic bias models, in which

$$
\rho_{\text {light }}=f\left(\rho_{\text {mass }}\right)+\epsilon,
$$

where $\epsilon$ is a random field that is uncorrelated with the mass density (Pen 1998; Dekel \& Lahav 1999). Although truly stochastic effects are possible in galaxy formation, a relation of the above form is expected when the galaxy and mass densities are filtered on some scale (as they always are, in practice). Just averaging a galaxy density that is a nonlinear function of

the mass will lead to some scatter when comparing with the averaged mass 

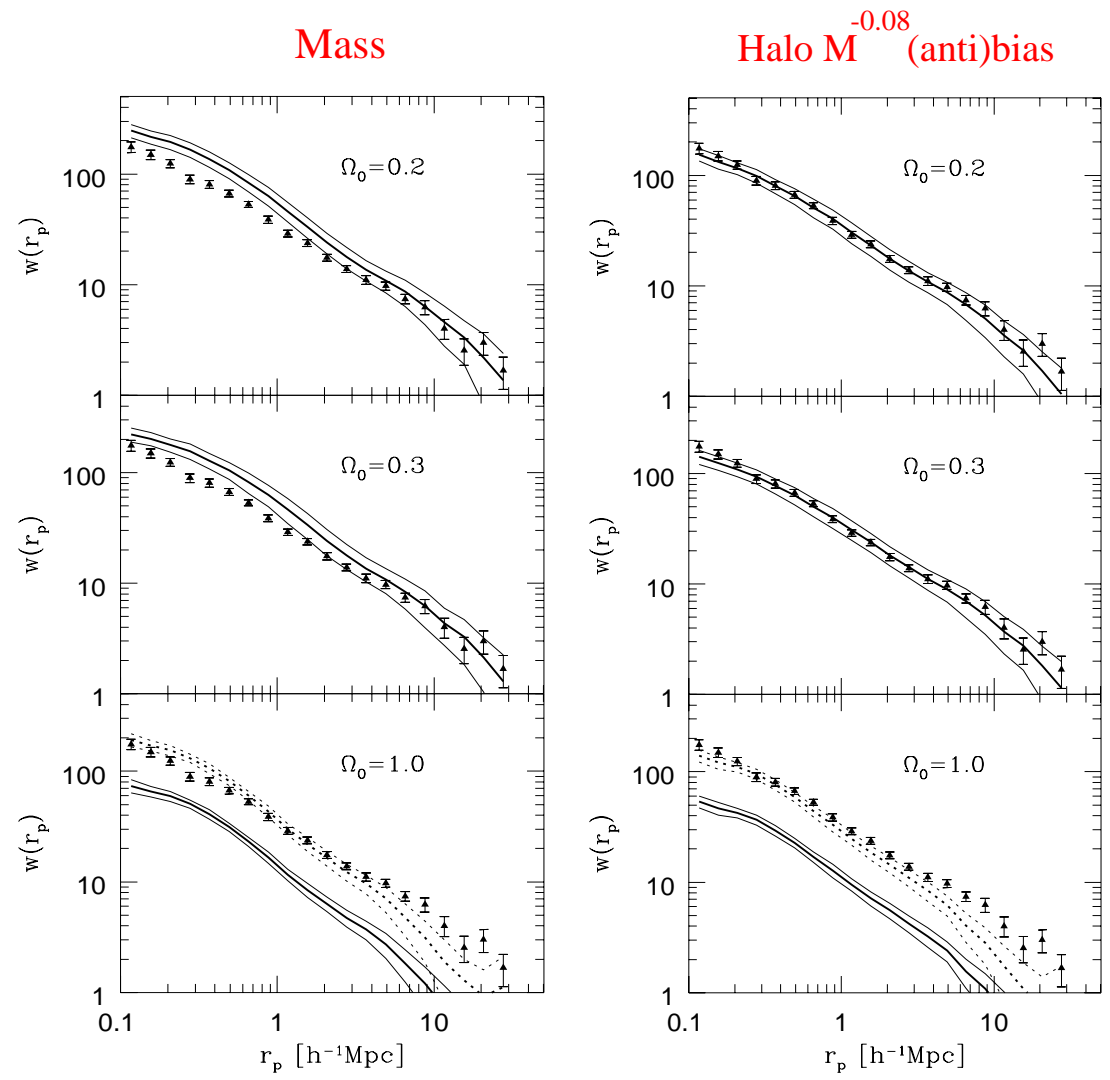

Figure 10. The projected correlation function from the LCRS fails to match CDM models when comparison is made to just the mass distribution. However, the agreement is excellent when allowance is made for a small degree of scale-dependent antibias; galaxy formation is suppressed in the most massive haloes (Jing, Mo \& Börner 1998).

field; a scatter will also arise when the relation between mass and light is non-local, however, and this may be the dominant effect.

The simplest and most important example of non-locality in the galaxyformation process is to recognize that galaxies will generally form where there are galaxy-scale haloes of dark matter. In the past, it was generally believed that dissipative processes were critically involved in galaxy formation, since pure collisionless evolution would lead to the destruction of galaxy-scale haloes when they are absorbed into the creation of a largerscale nonlinear system such as a group or cluster. However, it turns out that this overmerging problem was only an artefact of inadequate resolution. When a simulation is carried out with $\sim 10^{6}$ particles in a rich cluster, the cores of galaxy-scale haloes can still be identified after many crossing times (Ghigna et al. 1997). Furthermore, if catalogues of these 'sub-haloes' 
are created within a cosmological-sized simulation, their correlation function is quite different from that of the mass, resembling the single power law seen in galaxies (e.g. Klypin et al. 1999; Ma 1999).

These are very important results, and they hold out the hope that many of the issues concerning where galaxies form in the cosmic density field can be settled within the domain of collisionless simulations. Dissipative physics will still be needed to understand in detail the star-formation history within a galaxy-scale halo. Nevertheless, the idea that there may be a one-to-one correspondence between galaxies and galaxy-scale dark-matter haloes is clearly an enormous simplification - and one that increases the chance of making robust predictions of the statistical properties of the galaxy population.

\subsection{NUMERICAL GALAXY FORMATION}

The formation of galaxies must be a non-local process to some extent. The modern paradigm was introduced by White \& Rees (1978): galaxies form through the cooling of baryonic material in virialized haloes of dark matter. The virial radii of these systems are in excess of $0.1 \mathrm{Mpc}$, so there is the potential for large differences in the correlation properties of galaxies and dark matter on these scales.

A number of studies have indicated that the observed galaxy correlations may indeed be reproduced by CDM models. The most direct approach is a numerical simulation that includes gas, and relevant dissipative processes. This is challenging, but just starting to be feasible with current computing power (Pearce et al. 1999). The alternative is 'semianalytic' modelling, in which the merging history of dark-matter haloes is treated via the extended Press-Schechter theory (Bond et al. 1991), and the location of galaxies within haloes is estimated using dynamical-friction arguments (e.g. Cole et al. 1996; Kauffmann et al. 1996; Somerville \& Primack 1997). Both these approaches have yielded similar conclusions, and shown how CDM models can match the galaxy data: specifically, the low-density flat $\Lambda$ CDM model that is favoured on other grounds can yield a correlation function that is close to a single power law over $1000 \gtrsim \xi \gtrsim 1$, even though the mass correlations show a marked curvature over this range (Pearce et al. 1999; Benson et al. 1999; see figure 11). These results are impressive, yet it is frustrating to have a result of such fundamental importance emerge from a complicated calculational apparatus. There is thus some motivation for constructing a simpler heuristic model that captures the main processes at work in the full semianalytic models. The following section describes an approach of this sort (Peacock \& Smith, in preparation). 


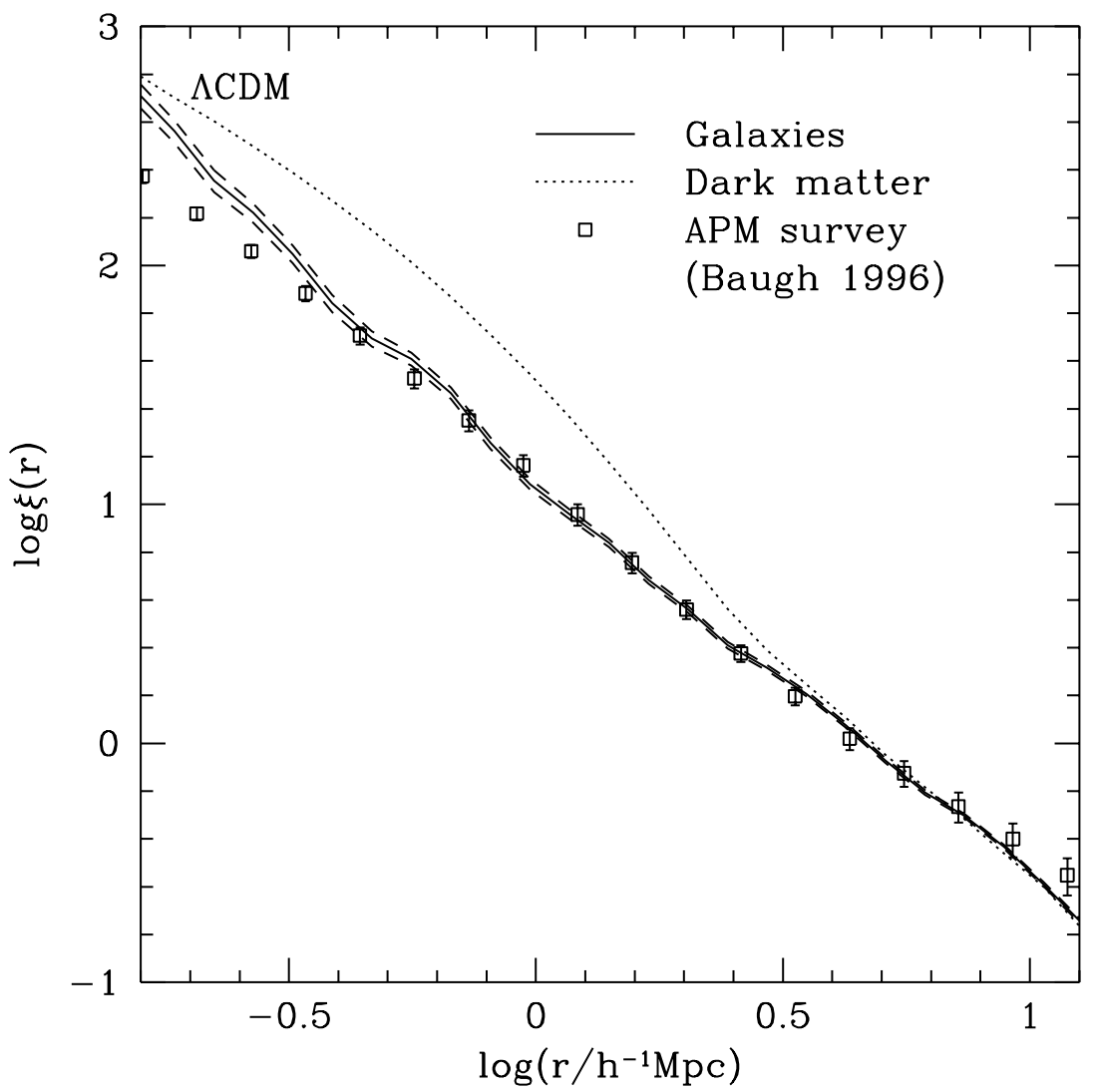

Figure 11. The correlation function of galaxies in the semianalytical simulation of an LCDM universe by Benson et al. (1999).

\subsection{HALO-OLOGY AND BIAS}

We mentioned above the early model of Neyman, Scott \& Shane (1953), in which the nonlinear density field was taken to be a superposition of randomly-placed clumps. With our present knowledge about the evolution of CDM universes, we can make this idealised model considerably more realistic: hierarchical models are expected to contain a distribution of masses of clumps, which have density profiles that are more complicated than isothermal spheres. These issues are well studied in $N$-body simulations, and highly accurate fitting formulae exist, both for the mass function and for the density profiles. Briefly, we use the mass function of Sheth \& Tormen 
(1999; ST) and the halo profiles of Moore et al. (1999; M99).

$$
\begin{aligned}
f(\nu) & =0.21617\left[1+\left(\sqrt{2} / \nu^{2}\right)^{0.3}\right] \exp \left[-\nu^{2} /(2 \sqrt{2})\right] \\
\Rightarrow F(>\nu) & =0.32218\left[1-\operatorname{erf}\left(\nu / 2^{3 / 4}\right)\right] \\
& +0.14765 \Gamma\left[0.2, \nu^{2} /(2 \sqrt{2})\right],
\end{aligned}
$$

where $\Gamma$ is the incomplete gamma function.

Recently, it has been claimed by Moore et al. (1999; M99) that the commonly-adopted density profile of Navarro, Frenk \& White (1996; NFW) is in error at small $r$. M99 proposed the alternative form

$$
\rho / \rho_{b}=\frac{\Delta_{c}}{y^{3 / 2}\left(1+y^{3 / 2}\right)} ; \quad\left(r<r_{\mathrm{vir}}\right) ; \quad y \equiv r / r_{c} .
$$

Using this model, it is then possible to calculate the correlations of the nonlinear density field, neglecting only the large-scale correlations in halo positions. The power spectrum determined in this way is shown in figure 12 , and turns out to agree very well with the exact nonlinear result on small and intermediate scales. The lesson here is that a good deal of the nonlinear correlations of the dark matter field can be understood as a distribution of random clumps, provided these are given the correct distribution of masses and mass-dependent density profiles.

How can we extend this model to understand how the clustering of galaxies can differ from that of the mass? There are two distinct ways in which a degree of bias is inevitable:

(1) Halo occupation numbers. For low-mass haloes, the probability of obtaining an $L^{*}$ galaxy must fall to zero. For haloes with mass above this lower limit, the number of galaxies will in general not scale with halo mass.

(2) Nonlocality. Galaxies can orbit within their host haloes, so the probability of forming a galaxy depends on the overall halo properties, not just the density at a point. Also, the galaxies will end up at special places within the haloes: for a halo containing only one galaxy, the galaxy will clearly mark the halo centre. In general, we expect one central galaxy and a number of satellites.

The numbers of galaxies that form in a halo of a given mass is the prime quantity that numerical models of galaxy formation aim to calculate. However, for a given assumed background cosmology, the answer may be determined empirically. Galaxy redshift surveys have been analyzed via grouping algorithms similar to the 'friends-of-friends' method widely employed to find virialized clumps in $N$-body simulations. With an appropriate correction for the survey limiting magnitude, the observed number of 


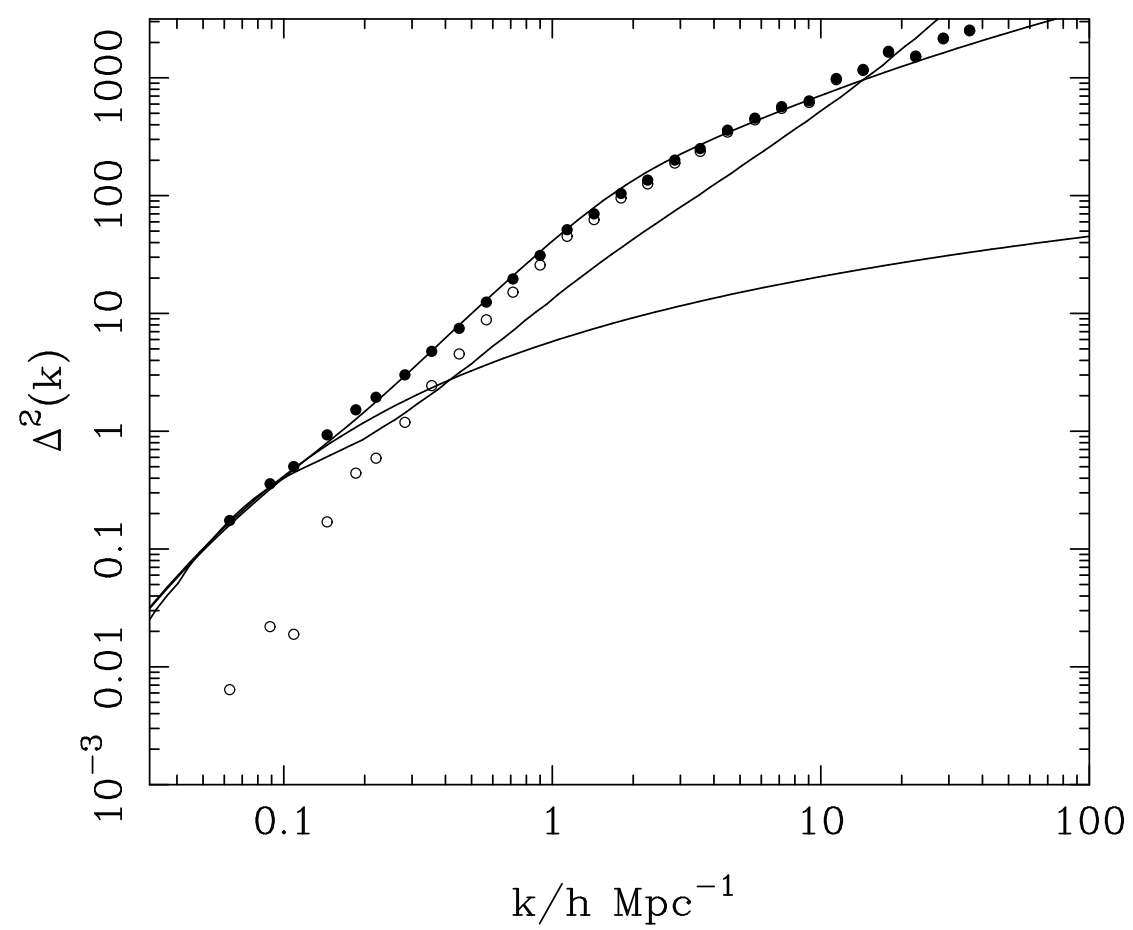

Figure 12. The power spectrum for the $\Lambda \mathrm{CDM}$ model. The solid lines contrast the linear spectrum with the nonlinear spectrum, calculated according to the approximation of PD96. The spectrum according to randomly-placed haloes is denoted by open circles; if the linear power spectrum is added, the main features of the nonlinear spectrum are well reproduced.

galaxies in a group can be converted to an estimate of the total stellar luminosity in a group. This allows a determination of the All Galaxy System (AGS) luminosity function: the distribution of virialized clumps of galaxies as a function of their total luminosity, from small systems like the Local Group to rich Abell clusters.

The AGS function for the CfA survey was investigated by Moore, Frenk \& White (1993), who found that the result in blue light was well described by

$$
d \phi=\phi^{*}\left[\left(L / L^{*}\right)^{\beta}+\left(L / L^{*}\right)^{\gamma}\right]^{-1} d L / L^{*},
$$

where $\phi^{*}=0.00126 h^{3} \mathrm{Mpc}^{-3}, \beta=1.34, \gamma=2.89$; the characteristic luminosity is $M^{*}=-21.42+5 \log _{10} h$ in Zwicky magnitudes, corresponding to $M_{B}^{*}=-21.71+5 \log _{10} h$, or $L^{*}=7.6 \times 10^{10} h^{-2} L_{\odot}$, assuming $M_{B}^{\odot}=5.48$. One notable feature of this function is that it is rather flat at low luminosities, in contrast to the mass function of dark-matter haloes (see Sheth 


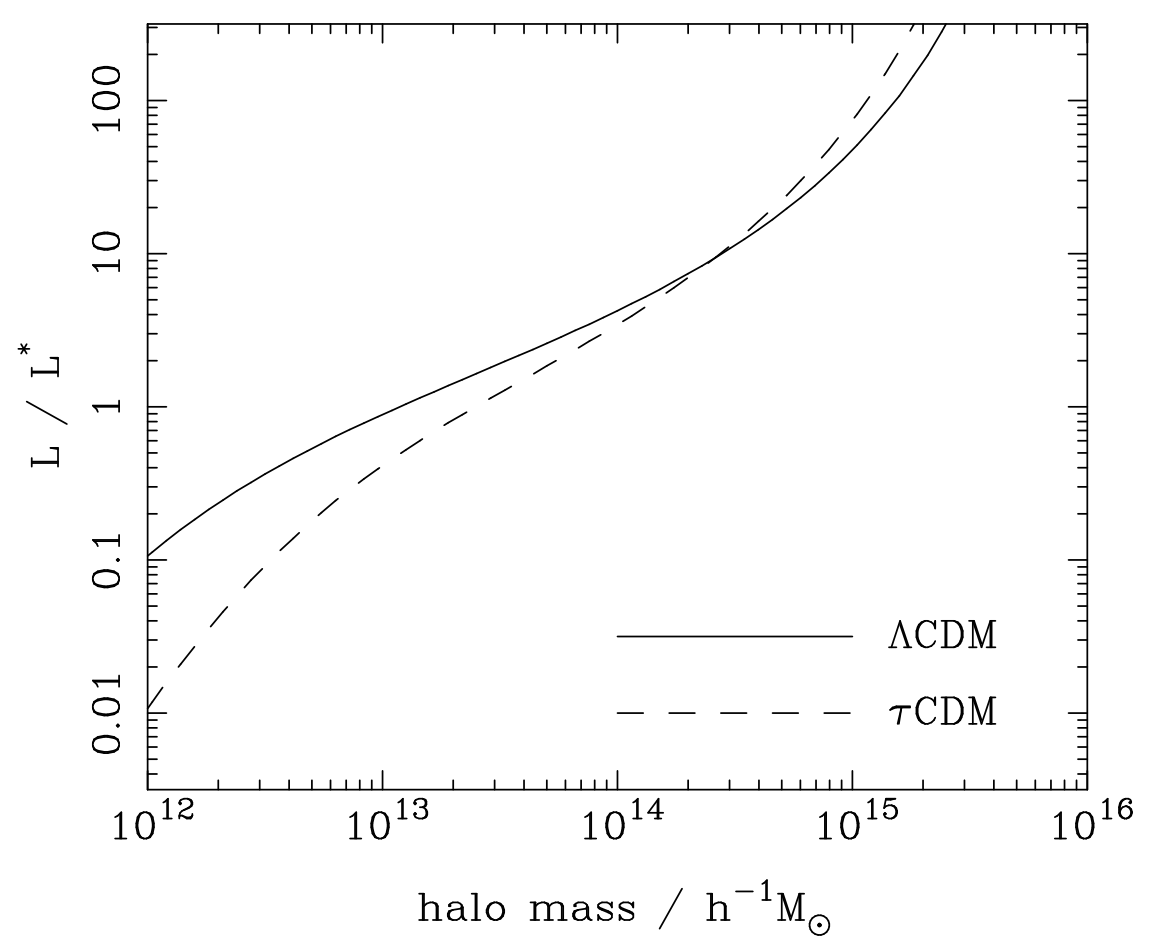

Figure 13. The empirical luminosity-mass relation required to reconcile the observed AGS luminosity function with two variants of CDM. $L^{*}$ is the characteristic luminosity in the AGS luminosity function $\left(L^{*}=7.6 \times 10^{10} h^{-2} L_{\odot}\right)$. Note the rather flat slope around $M=10^{13}$ to $10^{14} h^{-1} M_{\odot}$, especially for $\Lambda$ CDM.

\& Tormen 1999). It is therefore clear that any fictitious galaxy catalogue generated by randomly sampling the mass is unlikely to be a good match to observation. The simplest cure for this deficiency is to assume that the stellar luminosity per virialized halo is a monotonic, but nonlinear, function of halo mass. The required luminosity-mass relation is then easily deduced by finding the luminosity at which the integrated AGS density $\Phi(>L)$ matches the integrated number density of haloes with mass $>M$. The result is shown in figure 13.

We can now return to the halo-based galaxy power spectrum and use the correct occupation number, $N$, as a function of mass. This is needs a little care at small numbers, however, since the number of haloes with occupation number unity affects the correlation properties strongly. These haloes contribute no correlated pairs, so they simply dilute the signal from the haloes with $N \geq 2$. The existence of antibias on intermediate scales can probably be traced to the fact that a large fraction of galaxy groups contain only one $>L_{*}$ galaxy. Finally, we need to put the galaxies in the correct 


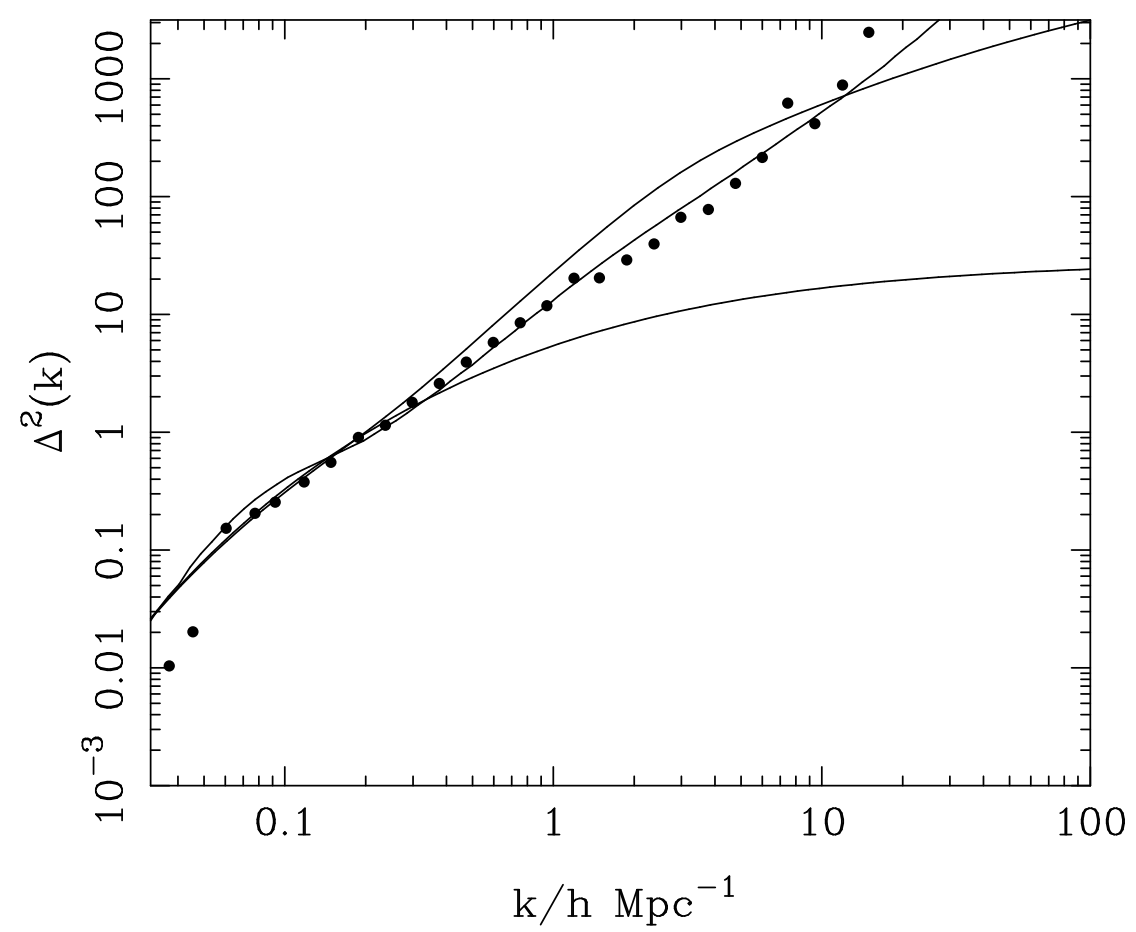

Figure 14. The power spectrum for a galaxy catalogue constructed from the $\Lambda$ CDM model. A reasonable agreement with the APM data (solid line) is achieved by simple empirical adjustment of the occupation number of galaxies as a function of halo mass, plus a scheme for placing the haloes non-randomly within the haloes.

location, as discussed above. If one galaxy always occupies the halo centre, with others acting as satellites, the small-scale correlations automatically follow the slope of the halo density profile, which keeps them steep. The results of this exercise are shown in figure 14 .

Although it is encouraging that it is possible to find simple models in which it is possible to understand the observed correlation properties of galaxies, there are other longstanding puzzles concerning the galaxy distribution. Arguably the chief of these concerns the dynamical properties of galaxies, in particular the pairwise peculiar velocity dispersion. This statistic has been the subject of debate, and preferred values have crept up in recent years, to perhaps 450 or $500 \mathrm{~km} \mathrm{~s}^{-1}$ at projected separations around 1 Mpc (e.g. Jing, Mo \& Börner 1998), most simple models predict a higher figure. Clearly, the amplitude of peculiar velocities depends on the normalization of the fluctuation spectrum; however, if this is set from the abundance of rich clusters, then Jenkins et al. (1998) found that reasonable values were predicted for large-scale streaming velocities, independent of $\Omega$. 
However, Jenkins et al. also found a robust prediction for the pairwise peculiar velocity dispersion around $1 \mathrm{Mpc}$ of about $800 \mathrm{~km} \mathrm{~s}^{-1}$. The observed galaxy velocity field appears to have a higher 'cosmic Mach number' than the predicted dark-matter distribution.

This difficulty is also solved by the simple bias model discussed here. Two factors contribute: the variation of occupation number with mass downweights the contribution of more massive groups, with larger velocity dispersions. Also, where one galaxy is centred on a halo, it gains a peculiar velocity which is that of the centre of mass of the halo, but does not reflect the internal velocity dispersion of the halo. Given a full $N$-body simulation, it is easy enough to predict what would be expected for a realistic bias model: one needs to construct a halo catalogue, calculating the peculiar velocities and internal velocity dispersions of each halo. Knowing the occupation number as a function of mass, a montecarlo catalogue of 'galaxies' complete with peculiar velocities can be generated. As shown in figure 15 , the effect of the empirical bias recipe advocated here is sufficient to reduce the predicted dispersion into agreement with observation. The simple model outlined here thus gives a consistent picture, and it is tempting to believe that it may capture some of the main features of realistic models for galaxy bias.

\section{Conclusions}

It should be clear from these lectures that large-scale structure has advanced enormously as a field in the past two decades. Many of our longstanding ambitions have been realised; in some cases, much faster than we might have expected. Of course, solutions for old problems generate new difficulties. We now have good measurements of the clustering spectrum and its evolution, and it is arguable that the discussion of section 7.4 captures the main features of the placement of galaxies with respect to the mass. However, a fairly safe bet is that one of the major results from new large surveys such as $2 \mathrm{dF}$ and Sloan will be a heightened appreciation of the subtleties of this problem.

Nevertheless, we should not be depressed if problems remain. Observationally, we are moving from an era of $20 \%$ - 50\% accuracy in measures of large-scale structure to a future of pinpoint precision. This maturing of the subject will demand more careful analysis and rejection of some of our existing tools and habits of working. The prize for rising to this challenge will be the ability to claim a real understanding of the development of structure in the universe. We are not there yet, but there is a real prospect that the next 5-10 years may see this remarkable goal achieved. 


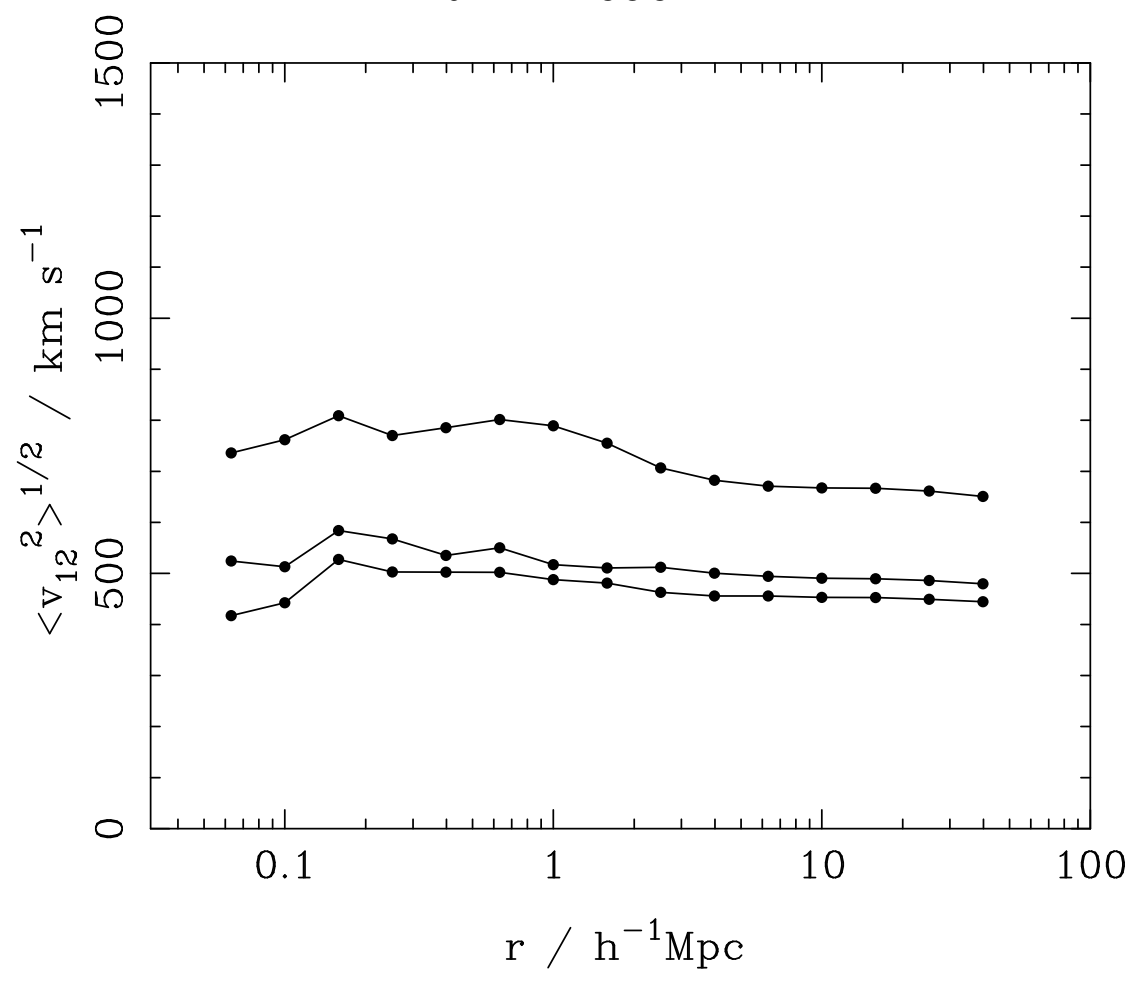

Figure 15. The line-of-sight pairwise velocity dispersion for the $\Lambda$ CDM model. The top curve shows the results for all the mass; the lower pair of curves shows the predicted galaxy results, with and without assuming that one galaxy occupies the halo centre (the former case gives the lowest curve).

\section{Acknowledgements}

I thank my colleagues in the $2 \mathrm{dF}$ Galaxy Redshift Survey for permission to reproduce our joint results in section 5.3, and Robert Smith for the joint work reported in section 7.4 .

\section{References}

Ballinger W.E., Peacock J.A., Heavens A.F., 1996, MNRAS, 282, 877

Baugh C.M., Efstathiou G., 1993, MNRAS, 265, 145

Baugh C.M., Efstathiou G., 1994, MNRAS, 267, 323

Benson A.J., Cole S., Frenk C.S., Baugh C.M., Lacey C.G., 1999, astro$\mathrm{ph} / 9903343$

Bond J.R., Cole S., Efstathiou G., Kaiser N., 1991, Astrophys. J., 379, 440

Bond J.R., 1995, Phys. Rev. Lett., 74, 4369

Bunn E.F., 1995, PhD thesis, Univ. of California, Berkeley

Coles P., 1993, MNRAS, 262, 1065 
Colless M., 1999, Phil. Trans. R. Soc. Lond. A, 357, 105

Davis M., Peebles P.J.E., 1983, Astrophys. J., 267, 465

Dekel A., Lahav O., 1999, Astrophys. J., 520, 24

Efstathiou G., Sutherland W., Maddox S.J., 1990, Nature, 348, 705

Eisenstein D.J., Hu W., 1998, ApJ, 496, 605

Eisenstein D.J., Zaldarriaga M., 1999, astro-ph/9912149

Eke V.R., Cole S., Frenk C.S., 1996, Mon. Not. R. Astr. Soc., 282, 263

Feldman H.A., Kaiser N., Peacock J.A., 1994, Astrophys. J., 426, 23

Folkes S., et al., 1999, Mon. Not. R. Astr. Soc., 308, 459

Gaztañaga E., Baugh C.M. 1998, Mon. Not. R. Astr. Soc., 294, 229

Ghigna S., Moore B., Governato F., Lake G., Quinn T., Stadel J., 1998, Mon. Not. R. Astr. Soc., 300, 146

Goldberg D.M., Strauss M.A., 1998, ApJ, 495, 29

Hamilton A.J.S., Kumar P., Lu E., Matthews A., 1991, Astrophys. J., 374, L1

Hamilton A.J.S., 1992, Astrophys. J., 385, L5

Hamilton A.J.S., 1997a, astro-ph/9708102

Hamilton A.J.S., 1997b, Mon. Not. R. Astr. Soc., 289, 285

Hamilton A.J.S., 1997c, Mon. Not. R. Astr. Soc., 289, 295

Huchra J.P., Geller M.J., de Lapparant V., Corwin H.G., 1990, Astrophys. J. Suppl., 72, 433

Jain B., Mo H.J., White S.D.M., 1995, Mon. Not. R. Astr. Soc., 276, L25

Jenkins A., Frenk C.S., Pearce F.R., Thomas P.A., Colberg J.M., White S.D.M., Couchman H.M.P., Peacock J.A., Efstathiou G., Nelson A.H., 1998, ApJ, 499, 20

Jing Y.P., Mo H.J., Börner G., 1998, ApJ, 494, 1

Kaiser N., 1987, MNRAS, 227, 1

Klypin A., Primack J., Holtzman J., 1996, Astrophys. J., 466, 13

Klypin A., Gottloeber S., Kravtsov A.V., Khokhlov A.M., 1999, 516, 530

Ma C.-P., 1999, Astrophys. J., 510, 32

Maddox S. Efstathiou G., Sutherland W.J., 1996, MNRAS, 283, 1227

Mann R.G., Peacock J.A., Heavens A.F., 1998, MNRAS, 293, 209

Margon B., 1999, Phil. Trans. R. Soc. Lond. A, 357, 93

Matsubara T., Szalay A.S., Landy S.D., 1999, astro-ph/9911151

Meiksin A.A., White M., Peacock J.A., MNRAS, 1999, 304, 851

Moore B., Frenk C.S., White S.D.M., 1993, Mon. Not. R. Astr. Soc., 261, 827

Moore B., Quinn T., Governato F., Stadel J., Lake G., 1999, astro-ph/9903164

Navarro J.F., Frenk C.S., White S.D.M., 1996, ApJ, 462, 563

Neyman, Scott \& Shane 1953, ApJ, 117, 92

Padmanabhan N., Tegmark M., Hamilton A.J.S., 1999, astro-ph/9911421

Peacock J.A., Dodds S.J., 1994, MNRAS, 267, 1020 
Peacock J.A., Dodds S.J., 1996, MNRAS, 280, L19

Peacock J.A., 1997, Mon. Not. R. Astr. Soc., 284, 885

Pearce F.R., et al., 1999, astro-ph/9905160

Peebles P.J.E., 1974, A\&A, 32, 197

Pen, W.-L., 1998, ApJ, 504, 601

Saunders W., et al., 1999, astro-ph/9909191

Shectman S.A., Landy S.D., Oemler A., Tucker D.L., Lin H., Kirshner R.P., Schechter P.L., 1996, Astrophys. J., 470, 172

Sheth R.K., Tormen G., 1999, astro-ph/9901122

Strauss M.A., Willick J.A., 1995, Physics Reports, 261, 271

Sugiyama N., 1995, Astrophys. J. Suppl., 100, 281

Tegmark M., 1996, Mon. Not. R. Astr. Soc., 280, 299

Tegmark M., Taylor A.N., Heavens A.F., 1997, Astrophys. J., 480, 22

Tegmark M., Hamilton A.J.S., Strauss M.A., Vogeley M.S., Szalay A.S., 1998, Astrophys. J., 499, 555

Viana P.T., Liddle A.R., 1996, MNRAS, 281, 323

Vogeley M.S., Szalay, A.S., 1996, Astrophys. J., 465, 34

White S.D.M., Rees M., 1978, Mon. Not. R. Astr. Soc., 183, 341

White S.D.M., Efstathiou G., Frenk C.S., 1993, Mon. Not. R. Astr. Soc., 262, 1023

Yano T., Gouda N., 1999, astro-ph/9906375 